\title{
Electron dose calculations using the Method of Moments
}

\author{
Edward W. Larsen \\ Department of Nuclear Engineering and Radiological Sciences, University of Michigan, \\ Ann Arbor, Michigan 48109
}

Moyed M. Miften ${ }^{\mathrm{a})}$ and Benedick A. Fraass

Department of Radiation Oncology, University of Michigan Medical Center, Ann Arbor, Michigan 48109

laïn A. D. Bruinvis

Department of Radiotherapy, The Netherlands Cancer Institute, Plesmanlaan 121, 1066 CX Amsterdam, The Netherlands

(Received 9 May 1996; accepted for publication 10 October 1996)

\begin{abstract}
The Method of Moments is generalized to predict the dose deposited by a prescribed source of electrons in a homogeneous medium. The essence of this method is (i) to determine, directly from the linear Boltzmann equation, the exact mean fluence, mean spatial displacements, and meansquared spatial displacements, as functions of energy; and (ii) to represent the fluence and dose distributions accurately using this information. Unlike the Fermi-Eyges theory, the Method of Moments is not limited to small-angle scattering and small angle of flight, nor does it require that all electrons at any specified depth $z$ have one specified energy $E(z)$. The sole approximation in the present application is that for each electron energy $E$, the scalar fluence is represented as a spatial Gaussian, whose moments agree with those of the linear Boltzmann solution. Numerical comparisons with Monte Carlo calculations show that the Method of Moments yields expressions for the depth-dose curve, radial dose profiles, and fluence that are significantly more accurate than those provided by the Fermi-Eyges theory. (C) 1997 American Association of Physicists in Medicine. [S0094-2405(97)00401-X]
\end{abstract}

Key words: electron beam, dose calculation, Gaussian, Method of Moments

\section{INTRODUCTION}

The problem of calculating dose is of basic importance in radiation oncology. Exact dose calculations require the solution of a coupled system of linear transport equations, to obtain the fluences for electrons and photons. This is an exceptionally difficult task, either from the mathematical or the computational perspective. Thus, clinical dose calculation algorithms typically rely on closed-form expressions that have their origins in analytic solutions of simplified problems, but contain empirically derived corrections to account for incomplete physics. This procedure yields clinical methods that are sufficiently fast for practical applications, but are limited in accuracy. In this paper, we present a new Method of Moments that partially bridges the gap between the expensive transport-based methods and the approximate clinical methods.

Currently, many clinical dose calculation algorithms, such as the pencil beam models proposed by Hogstrom et al., ${ }^{1}$ Brahme et al., ${ }^{2}$ and Werner et al., ${ }^{3}$ are based on the Fermi-Eyges ${ }^{4,5}$ theory. This underlying theory relies on approximations that commonly become invalid.

(i) Electrons are assumed to undergo only small-angle scattering in their interactions with the atoms of the medium. However, the neglect of large-angle scattering has a significant effect on the calculated dose distributions. ${ }^{6}$

(ii) Electrons are assumed to form a nearly monodirectional beam, i.e., to have a small angle of flight. At best, this is valid only for small energy loss (small depths). For large depths, electrons have undergone many collisions, and most have strayed far from their original direction of flight.

(iii) At any depth $z$, all electrons are assumed to have a specified energy $E(z)$. This is consistent with the approximations of small angle of flight and Continuous Slowing Down (CSDA) energy loss. However, these approximations neglect electron straggling, incorrectly equate pathlength with depth, and neglect large discrete energy loss due to secondary electron production (ionization).

In addition to these three basic assumptions, if one integrates the Fermi-Eyges solution over the spatial variables transverse to the direction of the beam, one obtains a constant, independent of depth. Thus, the Fermi-Eyges theory incorrectly equates the fluence with the planar fluence, so it cannot yield even qualitatively accurate depth dose curves.

For these reasons, the Fermi-Eyges solution is not directly used (without modification) in pencil beam models for clinical treatment planning. Typically, measured depth doses and other factors, some of which account for spatial inhomogeneities, are included to attempt to improve the accuracy of the overall solution. These improvements suffice for many applications, but not for others.

The pencil beam model and the underlying Fermi-Eyges theory have been studied extensively by many researchers. $^{7-10}$ Jette and Bielajew, ${ }^{11}$ Storchi and Huizenga, ${ }^{12}$ and Bruinvis et al. ${ }^{13}$ proposed ideas to deal with some of the limitations of the Fermi-Eyges theory. Shiu and Hogstrom ${ }^{14}$ proposed the Pencil Beam Redefinition model, and $\mathrm{Yu}$ 
et al. ${ }^{15}$ proposed the multi-ray model. These models are improvements over the pencil beam model, but they still use the Fermi-Eyges small-angle scattering theory.

A transport-based phase space evolution model, proposed by Huizenga and Storchi, ${ }^{16}$ yields accurate dose calculations in a reasonable time for broad electron beams. Monte Carlo methods have also been used for many years to simulate electron transport. Recent advances in computer hardware and in Monte Carlo algorithm development, such as the macro Monte Carlo method, ${ }^{17}$ are making Monte Carlo closer to a clinical treatment planning tool.

In this paper, we present an alternative Method of Moments (MM) for electron dose calculations in a homogeneous medium. This new method is based on the physically correct linear transport (or 'Boltzmann') equation. It generalizes earlier work (1950-1959) by Lewis, ${ }^{18}$ Spencer, Fano et al., ${ }^{19-22}$ and Kessaris. ${ }^{23}$ The Method of Moments has been used in the nuclear engineering community to predict gamma-ray and neutron penetration in shields ${ }^{24}$ and radiation damage in solids. ${ }^{25}$ In this paper, we present the Method of Moments as an approximate electron dose calculation algorithm that overcomes the approximations of the FermiEyges theory listed above, and that is less computationally intensive than algorithms based on the direct solution of the transport equation.

The Method of Moments in this paper consists of two parts.

(1) The exact space-angle moments of the fluence are calculated directly from the underlying transport equation. (See Appendix B. This procedure is algebraically equivalent to earlier work on the Method of Moments. ${ }^{18-25}$ )

(2) The spatial moments of the fluence are used to represent accurately the scalar fluence and the dose. (See Sec. II. We use a Gaussian spatial dependence for the fluence, unlike earlier work, which used non-Gaussian expansions.)

We show that with energy loss described by CSDA, the space-angle moments of the fluence can be obtained as explicit functions of electron energy $E$. If CSDA is not valid, the space-angle moments are the solution of a system of simple Volterra integral equations. In either case, the mean fluence, mean spatial displacements, and mean-squared spatial displacements are obtained explicitly in terms of the spatial moments of the fluence. Any computational steps needed to calculate the dose are simple and inexpensive. The sole approximation in this paper is that for each $E$, the scalar (angle-integrated) fluence is represented as a Gaussian function of the spatial coordinates, with energy-dependent amplitude, mean spatial displacements, and mean-squared spatial displacements, that are chosen to be equal to those of the transport solution.

The Method of Moments developed in this paper is conceptually the simplest in a hierarchy of approximations that would involve the calculation of higher-order spatial moments of the fluence and involve expressions that are more elaborate than a simple Gaussian. Nevertheless, for the simplest case of an initially monoenergetic, monodirectional beam with CSDA energy dependence (no secondary electrons), we show the following.
(1) Numerical comparisons of (i) the Method of Moments (MM) approximation of the dose, (ii) the Fermi-Eyges approximation, and (iii) Monte Carlo results in water, show that the MM expression gives reasonably accurate radial dose profiles and depth dose curves. Also, the MM results are more accurate than the Fermi-Eyges results, particularly for electrons at large depths.

(2) The angle-integrated MM solution satisfies a driftdiffusion equation with coefficients that depend on $E$ [see Eq. (D1)]. If small-angle scattering and small angular deflections are assumed, then this MM drift-diffusion equation reduces to the drift-diffusion equation satisfied by the FermiEyges solution. For large angular deflections, the MM driftdiffusion equation reduces to the Fermi-Age equation. ${ }^{26}$

An alternative derivation of some of the results in this paper, based on a stochastic model for electron transport, has recently been given. ${ }^{27}$ This stochastic model assumes that electrons lose their energy continuously as a function of pathlength, and change their direction at discrete "collisions" without losing any energy. The results of this analysis are identical to the results obtained here for electron transport with CSDA and no large-angle scattering. That is, the two results are identical for problems in which the standard Fokker-Planck approximation is valid.

The remainder of this paper is organized as follows. In Sec. II we describe the special "Boltzmann-CSD" transport problem and its explicit MM solution. In Sec. III we numerically compare the Method of Moments, Fermi-Eyges, and Monte Carlo solutions for 10 and $20 \mathrm{MeV}$ pencil beam problems. In Sec. IV we discuss our analytic and numerical results, and the relation between the Method of Moments and other schemes that perform electron dose calculations more accurately than the Fermi-Eyges theory.

Finally, in a set of Appendices we present the mathematical derivation of the Method of Moments. In Appendix A, we describe the Linear Boltzmann, Fokker-Planck, Boltzmann-Fokker-Planck, and Boltzmann-CSD Equations, each of which has been used to model electron transport. In Appendix B we derive the MM equations that determine space-angle moments of the fluence for each of these electron transport models. In Appendix $\mathrm{C}$ we obtain the $\mathrm{MM}$ system of first-order ordinary differential equations for the Boltzmann-CSD equation. (The explicit solutions of these equations are given in Sec. II.) In Appendix D, we demonstrate the connection between the MM-Boltzmann-CSD solution, the Fermi-Eyges solution, and the Fermi-Age solutions.

\section{STATEMENT OF THE PROBLEM AND THE METHOD OF MOMENTS SOLUTION}

We will now describe the MM solution for an electron transport problem with large-angle scattering and CSD energy dependence. This is probably the most physically realistic situation for which the space-angle moments of the fluence can be obtained in closed form. (In the Appendices, we describe how to treat more realistic problems, but we will not consider these further in this paper.) 
The "Boltzmann-CSD" (BCSD) transport equation with a point, monoenergetic, monodirectional source in an infinite homogeneous medium is

$\boldsymbol{\Omega} \cdot \boldsymbol{\nabla} \Phi(\mathbf{r}, \boldsymbol{\Omega}, E)+\Sigma_{t}(E) \Phi(\mathbf{r}, \boldsymbol{\Omega}, E)$

$$
\begin{aligned}
= & \int_{4 \pi} d \Omega^{\prime} \Sigma_{s}\left(E, \boldsymbol{\Omega} \cdot \boldsymbol{\Omega}^{\prime}\right) \Phi\left(\mathbf{r}, \boldsymbol{\Omega}^{\prime}, E\right)+\frac{\partial}{\partial E} S(E) \\
& \times \Phi(\mathbf{r}, \mathbf{\Omega}, E)+\frac{Q_{0}}{2 \pi} \delta(\mathbf{r}) \delta(\mu-1) \delta\left(E-E_{0}\right) .
\end{aligned}
$$

Here, $\mathbf{r}=(x, y, z)=\left(x_{1}, x_{2}, x_{3}\right)$ is the spatial variable, $\boldsymbol{\Omega}$ $=\left(\sqrt{1-\mu^{2}} \cos \phi, \sqrt{1-\mu^{2}} \sin \phi, \mu\right)=\left(\Omega_{1}, \Omega_{2}, \Omega_{3}\right)$ is the angular variable, $E$ is energy, $\Phi(\mathbf{r}, \boldsymbol{\Omega}, E)$ is the fluence, $S(E)$ is the stopping power, $\Sigma_{t}(E)$ is the total cross section, $\Sigma_{s}\left(E, \mu_{0}\right)$ is the differential scattering cross section, $\mu_{0}=\boldsymbol{\Omega}^{\prime} \cdot \mathbf{\Omega}=\cos \theta_{0}$, with $\theta_{0}$ the scattering angle, $Q_{0}$ is the amplitude of the point source, and $\delta$ is the Dirac delta function. The differential scattering cross section has the expansion

$$
\Sigma_{s}\left(E, \mu_{0}\right)=\sum_{n=0}^{\infty} \frac{2 n+1}{4 \pi} \Sigma_{s n}(E) P_{n}\left(\mu_{0}\right)
$$

where $P_{n}\left(\mu_{0}\right)$ is the $n$th Legendre polynomial. The expansion coefficients $\Sigma_{s n}$ in Eq. (2) are defined in terms of $\Sigma_{s}$ by

$$
\Sigma_{s n}(E)=2 \pi \int_{-1}^{1} d \mu_{0} P_{n}\left(\mu_{0}\right) \Sigma_{s}\left(E, \mu_{0}\right), \quad n \geqslant 0 .
$$

For electron transport, one has

$$
\begin{aligned}
& \Sigma_{t}(E)=\Sigma_{s 0}(E) \quad \text { (no absorption), } \\
& T(E) \equiv 2\left[\Sigma_{s 0}(E)-\Sigma_{s 1}(E)\right]=\text { scattering power. }
\end{aligned}
$$

To describe the MM solution, let us define the infinitemedium space-angle integration operator,

$$
\langle\Phi\rangle(E) \equiv \int d^{3} r \int d^{2} \Omega \Phi(\mathbf{r}, \mathbf{\Omega}, E) .
$$

Also, let us define the functions

$$
\begin{aligned}
& \Sigma_{a n}(E) \equiv \Sigma_{s 0}(E)-\Sigma_{s n}(E), \\
& f_{n}(E) \equiv \int_{E}^{E_{0}} d E^{\prime} \frac{\Sigma_{a n}\left(E^{\prime}\right)}{S\left(E^{\prime}\right)}, \quad n=1,2 .
\end{aligned}
$$

Then $T(E)=2 \Sigma_{a 1}(E)$. Manipulating Eqs. (1)-(7), as described in Appendices B and C, we obtain the following closed-form results for $0<E<E_{0}$ :

$$
\begin{aligned}
& \langle\Phi\rangle(E)=\frac{Q_{0}}{S(E)}, \\
& \langle\mu \Phi\rangle(E)=\langle\Phi\rangle(E) e^{-f_{1}(E)}, \\
& \langle z \Phi\rangle(E)=\frac{1}{S(E)} \int_{E}^{E_{0}} d E^{\prime}\langle\mu \Phi\rangle\left(E^{\prime}\right), \\
& \left\langle\mu^{2} \Phi\right\rangle(E)=\frac{1}{3}\langle\Phi\rangle(E)\left(1+2 e^{-f_{2}(E)}\right),
\end{aligned}
$$

$$
\begin{aligned}
& \left\langle x \cos \phi \sqrt{1-\mu^{2}} \Phi\right\rangle(E)=\frac{e^{-f_{1}(E)}}{2 S(E)} \int_{E}^{E_{0}} d E^{\prime}\left[\langle\Phi\rangle\left(E^{\prime}\right)\right. \\
& \left.-\left\langle\mu^{2} \Phi\right\rangle\left(E^{\prime}\right)\right] e^{f_{1}\left(E^{\prime}\right)}, \\
& \left\langle x^{2} \Phi\right\rangle(E)=\left\langle y^{2} \Phi\right\rangle(E) \\
& =\frac{2}{S(E)} \int_{E}^{E_{0}} d E^{\prime}\left\langle\sqrt{1-\mu^{2}}(\cos \phi) x \Phi\right\rangle\left(E^{\prime}\right), \\
& \langle\mu z \Phi\rangle(E)=\frac{e^{-f_{1}(E)}}{S(E)} \int_{E}^{E_{0}} d E^{\prime}\left\langle\mu^{2} \Phi\right\rangle\left(E^{\prime}\right) e^{f_{1}\left(E^{\prime}\right)}, \\
& \left\langle z^{2} \Phi\right\rangle(E)=\frac{2}{S(E)} \int_{E}^{E_{0}} d E^{\prime}\langle\mu z \Phi\rangle\left(E^{\prime}\right) .
\end{aligned}
$$

Also,

$$
\begin{aligned}
\langle x \Phi\rangle(E) & =\langle y \Phi\rangle(E)=\langle x y \Phi\rangle(E)=\langle y z \Phi\rangle(E) \\
& =\langle x z \Phi\rangle(E)=\langle z x \Phi\rangle(E)=0 .
\end{aligned}
$$

The mean particle displacements are defined by

$$
\begin{aligned}
& \bar{x}(E) \equiv \frac{\langle x \Phi\rangle(E)}{\langle\Phi\rangle(E)}=0, \\
& \bar{y}(E) \equiv \frac{\langle y \Phi\rangle(E)}{\langle\Phi\rangle(E)}=0, \\
& \bar{z}(E) \equiv \frac{\langle z \Phi\rangle(E)}{\langle\Phi\rangle(E)}=\int_{E}^{E_{0}} d E^{\prime} \frac{e^{-f_{1}\left(E^{\prime}\right)}}{S\left(E^{\prime}\right)} .
\end{aligned}
$$

Thus, the mean particle motion is along the $z$ axis. As $E$ decreases from $E_{0}$ to $0, \bar{z}(E)$ increases from 0 to a finite value, which is less than the electron range unless $T=0$. Of course, individual electrons may increasingly stray from this mean position as $E$ decreases. Therefore, the variances in particle positions should all increase as $E$ decreases.

These variances are defined by

$$
\begin{aligned}
& \sigma_{x}^{2}(E)=\sigma_{y}^{2}(E)=\frac{\left\langle x^{2} \Phi\right\rangle(E)}{\langle\Phi\rangle(E)} \equiv \sigma_{r}^{2}(E), \\
& \sigma_{z}^{2}(E)=\frac{\left\langle[z-\bar{z}(E)]^{2} \Phi\right\rangle(E)}{\langle\Phi\rangle(E)}=\frac{\left\langle z^{2} \Phi\right\rangle(E)}{\langle\Phi\rangle(E)}-\left(\frac{\langle z \Phi\rangle(E)}{\langle\Phi\rangle(E)}\right)^{2}
\end{aligned}
$$

with $\sigma_{x y}(E)=\sigma_{y z}(E)=\sigma_{z x}(E)=0$.

In Eqs. 16-20, the mean particle displacements and variances for each $E$ are explicitly given in terms of the spatial moments $\langle\Phi\rangle(E),\langle x \Phi\rangle(E),\left\langle x^{2} \Phi\right\rangle(E)$, and $\left\langle z^{2} \Phi\right\rangle(E)$.

Now let us define the function $F(\mathbf{r}, E)$ as

$$
\begin{aligned}
F(\mathbf{r}, E) \equiv & \langle\Phi\rangle(E)\left[\frac{1}{2 \pi \sigma_{r}^{2}(E)} \exp \left(-\frac{x^{2}+y^{2}}{2 \sigma_{r}^{2}(E)}\right)\right] \\
& \times\left[\frac{1}{\sqrt{2 \pi} \sigma_{z}(E)} \exp \left(-\frac{[z-\bar{z}(E)]^{2}}{2 \sigma_{z}^{2}(E)}\right)\right] .
\end{aligned}
$$

We have

$$
\int d^{3} r F(\mathbf{r}, E)=\langle\Phi\rangle(E),
$$




$$
\begin{aligned}
& \int d^{3} r z F(\mathbf{r}, E)=\langle z \Phi\rangle(E), \\
& \int d^{3} r x^{2} F(\mathbf{r}, E)=\int d^{3} r y^{2} F(\mathbf{r}, E)=\left\langle x^{2} \Phi\right\rangle(E), \\
& \int d^{3} r z^{2} F(\mathbf{r}, E)=\left\langle z^{2} \Phi\right\rangle(E),
\end{aligned}
$$

and

$$
\begin{aligned}
& \int d^{3} r x F(\mathbf{r}, E) \\
& =\int d^{3} r y F(\mathbf{r}, E) \\
& =\int d^{3} r x y F(\mathbf{r}, E) \\
& =\int d^{3} r y z F(\mathbf{r}, E)=\int d^{3} r z x F(\mathbf{r}, E)=0 .
\end{aligned}
$$

Thus, $F(\mathbf{r}, E)$ is a Gaussian in $\mathbf{r}$, whose energy-dependent mean value, mean spatial displacements, and mean-squared spatial displacements agree exactly with those of the angularly integrated solution of Eq. (1), for all $E$. We now approximate the true angularly integrated fluence by $F(\mathbf{r}, E)$ and obtain the following approximation for the dose:

$$
D(\mathbf{r})=\frac{1}{\rho} \int_{0}^{E_{0}} d E S(E) F(\mathbf{r}, E) .
$$

The depth-dose curve for an infinitely broad beam is defined by

$$
D_{\infty}(z) \equiv \int_{-\infty}^{\infty} d x \int_{-\infty}^{\infty} d y D(\mathbf{r})=\frac{1}{\rho} \int_{0}^{E_{0}} d E S(E) F_{\infty}(z, E),
$$

where

$$
\begin{aligned}
F_{\infty}(z, E) & \equiv \int_{-\infty}^{\infty} d x \int_{-\infty}^{\infty} d y F(\mathbf{r}, E) \\
& =\frac{\langle\Phi\rangle(E)}{\sqrt{2 \pi} \sigma_{x}(E)} \exp \left(-\frac{[z-\bar{z}(E)]^{2}}{2 \sigma_{x}^{2}(E)}\right) .
\end{aligned}
$$

The radial dose profile is defined by

$$
D_{r p}(x, y, z)=\frac{D(x, y, z)}{D(0,0, z)} .
$$

Equations (8)-(21) and (27) describe the MM solution of Eq. (1). Next, we shall compare numerically the MMBCSD, MM-Fokker-Planck, Fermi-Eyges, and Monte Carlo depth-dose and radial dose profiles for two electron pencil beam problems. We show in Appendix D that the MM-Fokker-Planck result is obtained from the results in this section by setting

$$
\Sigma_{a 2}(E)=3 \Sigma_{a 1}(E)=\frac{3}{2} T(E) .
$$

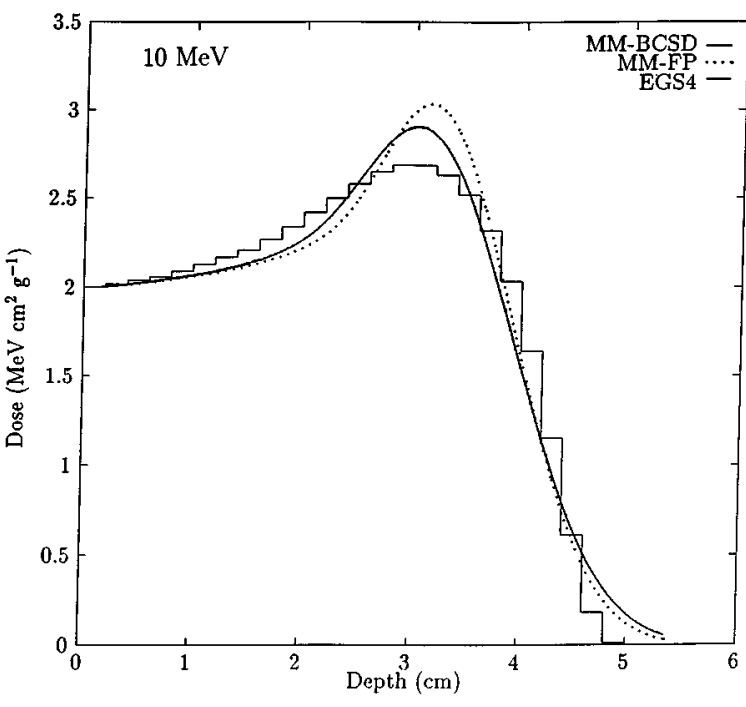

FIG. 1. Depth dose curve for a broad $10 \mathrm{MeV}$ electron beam.

\section{RESULTS}

Here we consider 10 and $20 \mathrm{MeV}$ electron pencil beams normally incident on a water phantom. We simulated these problems four ways: (i) the Method of Moments applied to the Boltzmann-CSD equation; (ii) the Method of Moments applied to the Fokker-Planck approximation to the Boltzmann-CSD equation (in which large-angle scattering is neglected); (iii) the Fermi-Eyges (FE) method; and (iv) Monte Carlo, using the EGS4 code ${ }^{28}$ with the PRESTA algorithm. ${ }^{29}$ In our Monte Carlo simulations we used $10^{6}$ electrons, the CSD approximation (no secondary electrons) with the unrestricted collision stopping power, and energy cutoffs set to $\mathrm{AE}$ $=\mathrm{ECUT}=0.521 \mathrm{MeV}$ and $\mathrm{AP}=\mathrm{PCUT}=0.01 \mathrm{MeV}$. To perform the MM and Fermi-Eyges calculations, we used a set of multigroup cross sections generated by the CEPXS code. ${ }^{30}$ Because of this multigroup approximation, there are discrepencies between the MM and EGS calculations. However, these discrepencies are slight.

Figures 1 and 2 show the broad-beam depth doses, as defined by Eqs. (28) and (29), for the MM-BCSD, the MMFP, and the EGS4 simulations. We see that the shapes of the two MM simulations are globally correct. The differences between the Monte Carlo and the MM-BCSD results are due to the assumption of a spatial Gaussian, while the differences between the Monte Carlo and the MM-FP results are due to the spatial Gaussian assumption and the small angle of scattering approximation. For these problems, the omission of large-angle scattering in the MM-FP simulation leads to an error in the depth-dose distribution that is approximately double that of the MM-BCSD simulation.

Actually, there is a second error in the MM-BCSD and MM-FP simulations. The Method of Moments developed in this paper strictly holds for an infinite homogeneous medium. However, here we have applied this method to the problem of a pencil beam normally incident on a semiinfinite water phantom. We have done this by approximating the semi-infinite phantom by an infinite phantom with a 


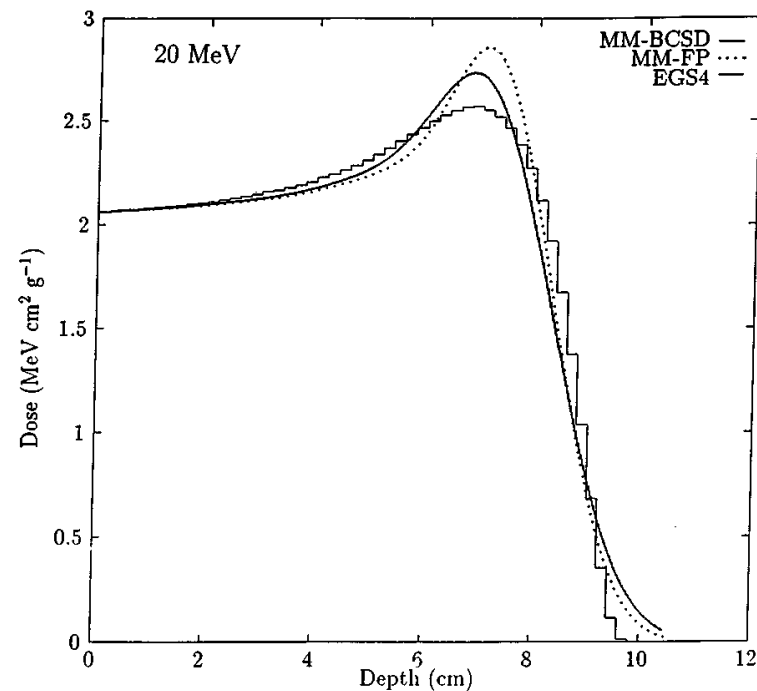

FIG. 2. Depth dose curve for a broad $20 \mathrm{MeV}$ electron beam.

monodirectional point source, and applying the Method of Moments to the latter problem. Since virtually none of the radiation backscatters out of the semi-infinite half-space $z>0$ the error resulting from approximating the semi-infinite phantom by an infinite phantom is negligible (much less than $1 \%)$.

In Figs. 3 and 4, radial dose profiles are plotted from the MM-BCSD, Fermi-Eyges, and EGS4 simulations at different depths. Near the central axis, the MM-BCSD and Fermi-Eyges profiles are remarkably similar. Away from the central axis, the MM-BCSD results are consistently greater than the Fermi-Eyges results, and agree with the Monte Carlo results over a larger range. The MM-FP profiles are very similar to the other profiles near the central axis; away from the central axis, they are closer to the MM-BCSD results than to the Fermi-Eyges results.

In Figs. 5 and 6, we plot as functions of the electron

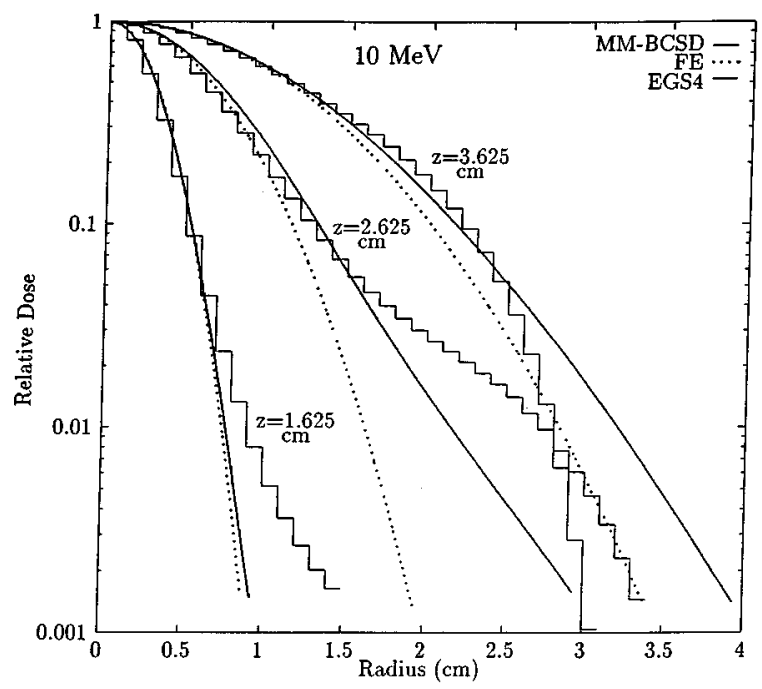

FIG. 3. Radial dose profiles for a $10 \mathrm{MeV}$ electron pencil beam.

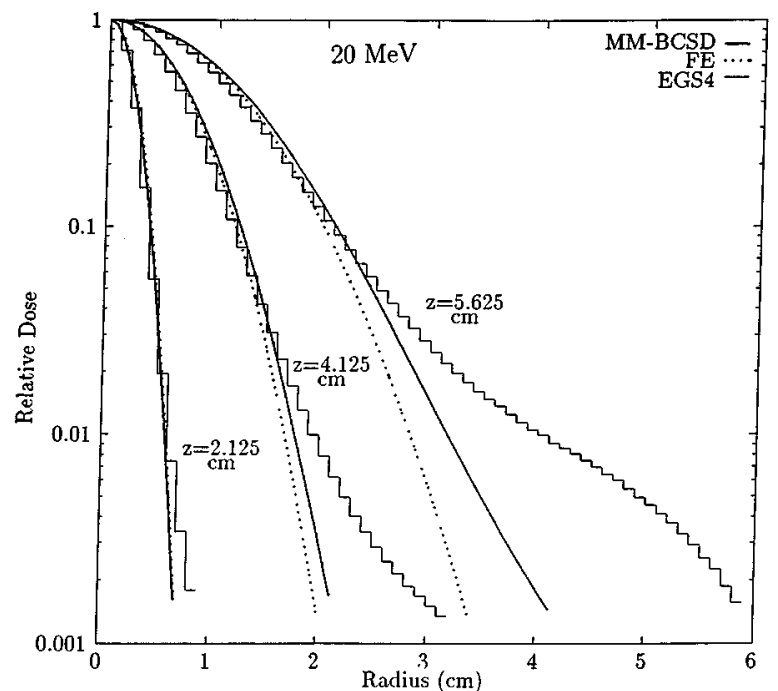

FIG. 4. Radial dose profiles for a $20 \mathrm{MeV}$ electron pencil beam.

energy $E \bar{z}(E), \sigma_{r}(E), \sigma_{z}(E)$ [see Eq. (21)], and the mean cosine of the angle of flight, defined as

$$
\bar{\mu}(E) \equiv \frac{\langle\mu \Phi\rangle(E)}{\langle\Phi\rangle(E)}=\exp \left(-\int_{E}^{E_{0}} d E^{\prime} \frac{T\left(E^{\prime}\right)}{2 S\left(E^{\prime}\right)}\right),
$$

for the 10 and $20 \mathrm{MeV}$ BCSD pencil beams. These plots show that $\bar{\mu}(E)$ deviates significantly from its initial value $(\theta=0, \bar{\mu}=1)$ as the electrons lose energy. Thus, over a substantial range of the electron energies, the small angle-offlight assumption of the Fermi-Eyges approximation is invalid. Also, as the electrons lose energy, $\sigma_{z}(E), \sigma_{r}(E)$, and $\bar{z}(E)$ monotonically increase, with $\sigma_{z}(E)<\sigma_{r}(E) \ll \bar{z}(E)$. Thus, the electrons are clustered around their mean position (on the central axis), with average deviations $\sigma_{z}(E), \sigma_{r}(E)$ from this mean position that grow with energy loss, but that remain small compared to the mean electron depth $\bar{z}(E)$.

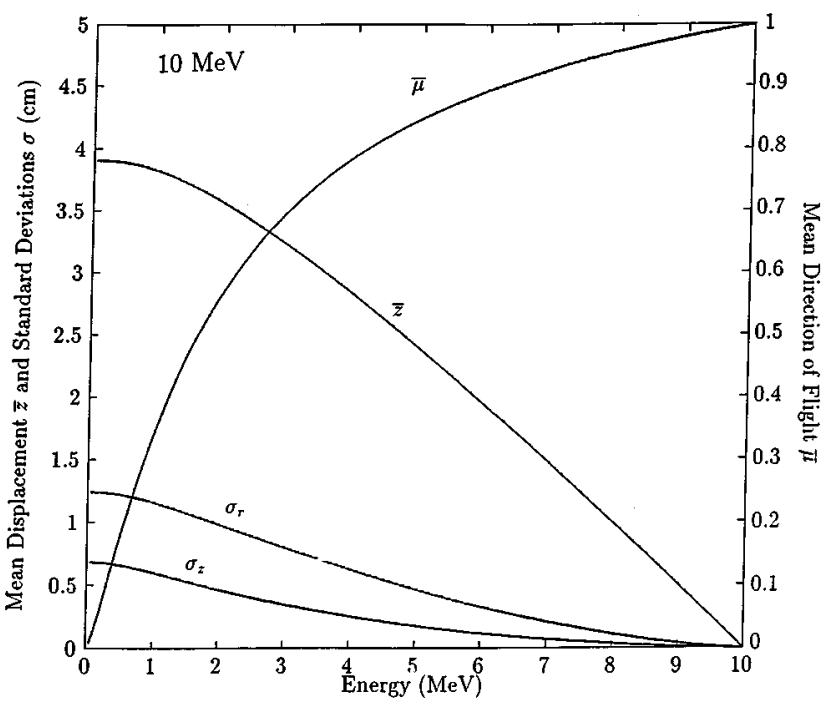

FIG. 5. We see $\sigma_{r}, \sigma_{z}, \bar{z}$, and $\bar{\mu}$ for a $10 \mathrm{MeV}$ electron pencil beam (BCSD equation). 


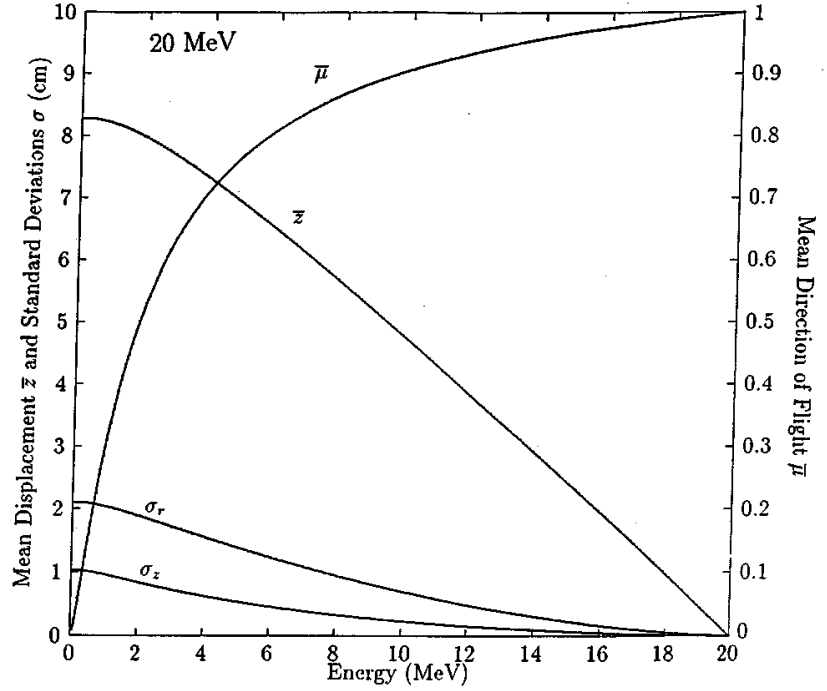

FIG. 6. We see $\sigma_{r}, \sigma_{z}, \bar{z}$, and $\bar{\mu}$ for a $20 \mathrm{MeV}$ electron pencil beam (BCSD equation).

In Fig. 7, we plot the aspect ratio $\sigma_{z}(E) / \sigma_{r}(E)$, for the $\mathrm{BCSD}$ and FP solutions of the $20 \mathrm{MeV}$ beam problem. Here we see a significant difference between the two solutions: the aspect ratio of the BCSD simulation increases from 0.27 for $20 \mathrm{MeV}$ to 0.49 for $0 \mathrm{MeV}$, while the FP approximation produces an aspect ratio of 0 for $20 \mathrm{MeV}$, increasing to 0.42 for $0 \mathrm{MeV}$. The aspect ratios of the $10 \mathrm{MeV}$ beam are similar and are not shown for brevity.

Thus, in the FP simulations, electrons with specific energies $E \approx E_{0}$ are distributed in an exceedingly narrow interval in $z$ around the mean depth $\bar{z}(E)$. (This is consistent with the Fermi-Eyges picture, in which all electrons at a specific energy $E$ exist at a single depth $z$.) However, in the BCSD solution, $\sigma_{z}$ does not become arbitrarily small in comparison

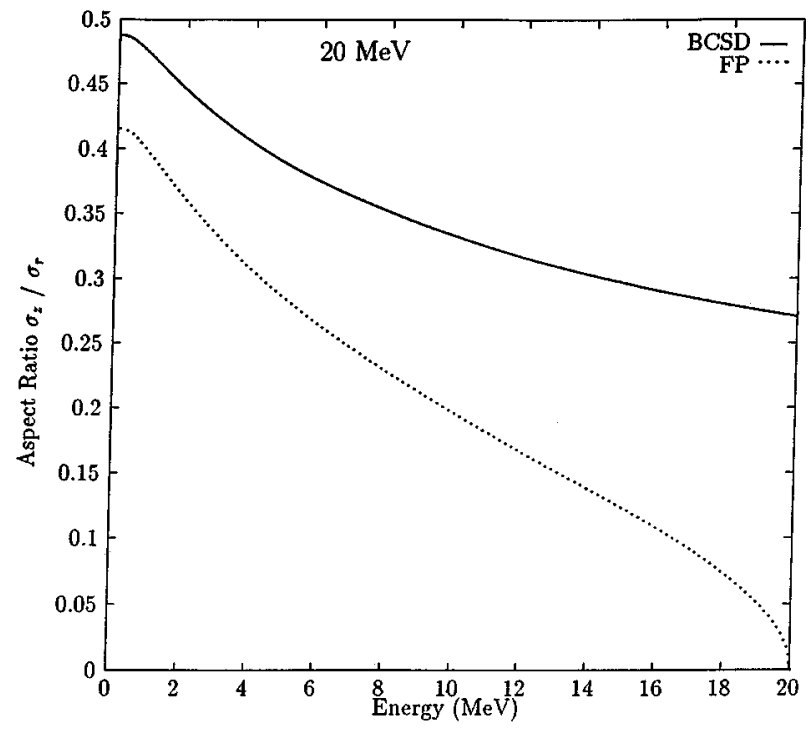

FIG. 7. The aspect ratio $\sigma_{r} / \sigma_{z}$ for a $20 \mathrm{MeV}$ electron pencil beam (BCSD and FP equations).

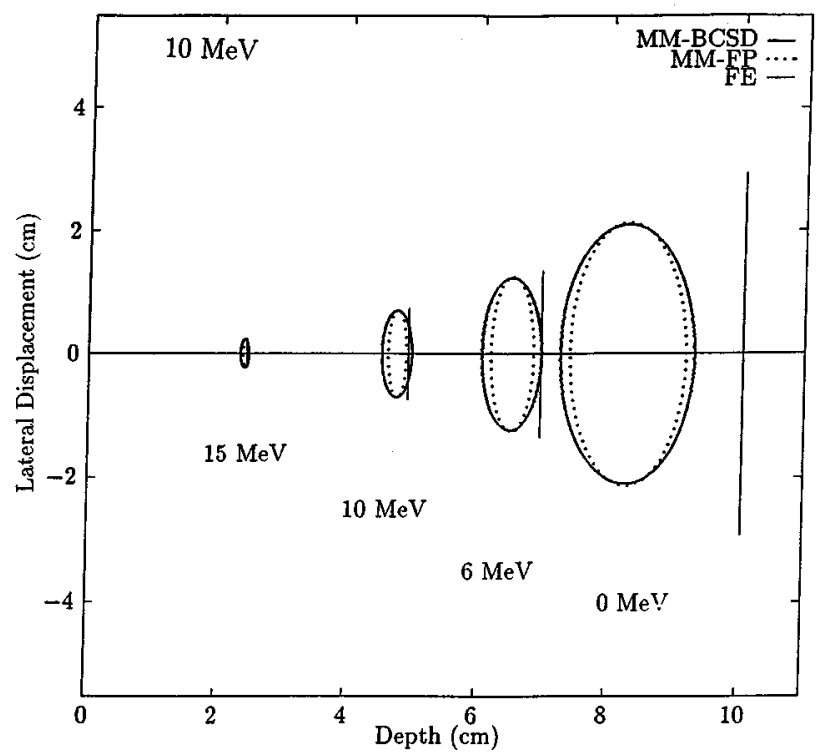

FIG. 8. Spatial distribution of electrons from a $20 \mathrm{MeV}$ pencil beam (MM$\mathrm{BCSD}, \mathrm{MM}-\mathrm{FP}$, and FE solutions).

to $\sigma_{r}$. Thus, while the mean displacements $\bar{z}(E)$ of the FP and BCSD solutions are identical, the presence of largeangle scattering in the BCSD equation creates a fundamentally different picture of electron positions about this mean displacement for $E \approx E_{0}$.

However, these differences may have little consequence in dose calculations; they are most significant for small energy loss and small depths, where the pencil beam is exceedingly narrow, and where the dose is likely to be deposited in the same voxel regardless of whether one uses the FP or the BCSD equation to describe the electron transport.

In Fig. 8, we plot data corresponding to various solutions for the $20 \mathrm{MeV}$ pencil beam at $15,10,6$, and $0 \mathrm{MeV}$. For the MM-BCSD and MM-FP solutions we plot ellipses, whose centers are at the points $\bar{z}(E)$, with minor axes $\sigma_{z}(E)$ and major axes $\sigma_{r}(E)$; electrons that lie in these ellipses are less than one standard deviation away from the mean displacement. The Fermi-Eyges solution yields vertical line segments, since in this theory all electrons at a specified energy exist at a single depth.

For small energy losses, the three methods all locate the electrons in roughly the same spatial region. For large energy losses, the Fermi-Eyges solution is exceedingly inaccurate; it places the electrons much deeper inside the phantom than they should be, and the assumption $E=E(z)$ is noticeably incorrect. The FP data for large energy loss is much closer to the BCSD data; the mean displacements of the two solutions are identical (as they should be; see Appendix C), and the mean-squared displacements agree to within about $10 \%$.

In Figs. 9 and 10, we plot the data of Fig. 8 for the MM-BCSD and Fermi-Eyges solutions, together with Monte Carlo results (500 electrons), for the 10 and $20 \mathrm{MeV}$ pencil beams. Slight discrepencies exist in the mean depth and variances between the EGS and the MM-BCSD results, due to the approximate multigroup cross sections used in the 


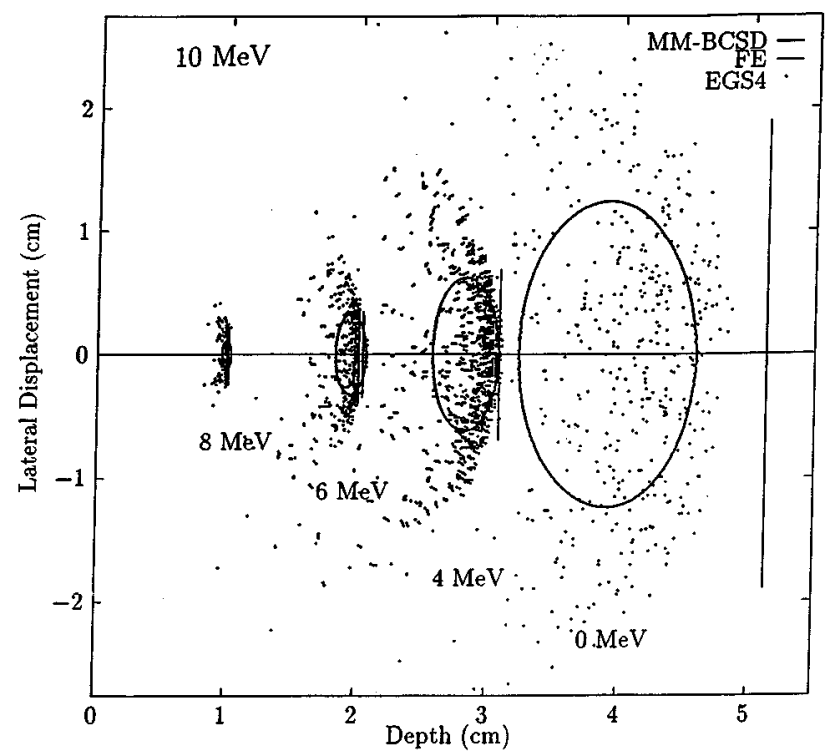

FIG. 9. Spatial distribution of electrons from a $10 \mathrm{MeV}$ pencil beam (MMBCSD, FE, and EGS4 solutions).

Method of Moments, and the continuous energy-dependent cross sections used in EGS. Nevertheless, these figures confirm that the MM-BCSD description of the fluence is much more physically realistic than the Fermi-Eyges solution. Also, Figs. 9 and 10 show that the spatial-Gaussian approximation made in this paper is not exact; the level curves of the scalar fluence are "umbrella shaped" around the mean displacements, rather than elliptically shaped. Thus, a suitable non-Gaussian ansatz could produce more accurate results.

\section{DISCUSSION}

We have presented the Method of Moments for calculating the dose due to a prescribed source of electrons in a

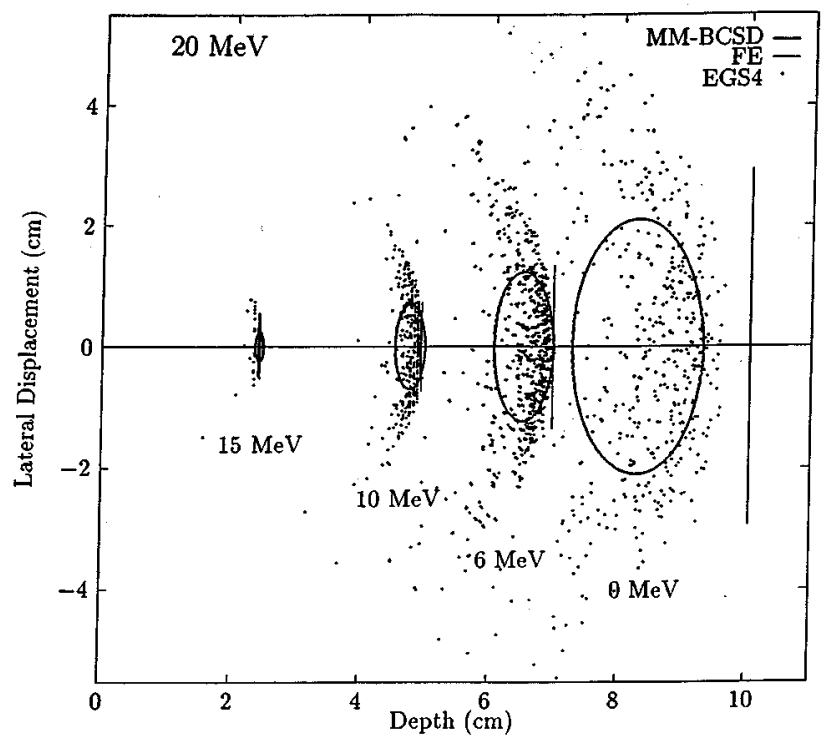

FIG. 10. Spatial distribution of electrons from a $20 \mathrm{MeV}$ pencil beam (MMBCSD, FE, and EGS4 solutions). homogeneous medium. This method is based on manipulating the costly-to-solve linear transport equation for the fluence into an easily solvable system of equations for the space-angle moments of the fluence. After the low-order spatial moments are determined, we represent the fluence by a spatial Gaussian with the same mean displacements and variances as the transport solution, and we use this approximation to calculate the dose. The Method of Moments does not require the small angle of scattering, small angle of flight, or $E=E(z)$ assumptions that are necessary for the FermiEyges theory. Our sole approximation is that for each energy $E$, the scalar fluence is represented as a Gaussian in $\mathbf{r}$.

For small depths, the radial dose distribution of a pencil beam from the Gaussian model based on the Fermi-Eyges theory overestimates the dose at small radii and underestimates the dose at large radii. This is because the FermiEyges model does not account for the few important largeangle scattering events. For large depths, where large-angle scattering events are relatively less important, the FermiEyges distribution overestimates the dose because the model incorrectly equates pathlength with depth.

The theory presented in this paper provides an answer to the question: What is the limit of accuracy of the Gaussian model for electron transport? That is, if one were able to determine the best possible Gaussian fit to the electron fluence, how accurately could one model the fluence and calculate the dose? We assert that the best Gaussian fit is one that, for each energy $E$, has mean spatial displacements and variances that are identical to those of the exact Boltzmann solution. This is precisely the MM approximation developed in this paper.

To understand how the Method of Moments solution relates to other schemes, such as the Phase Space Evolution (PSE) model ${ }^{16}$ or the Pencil Beam Redefinition model, ${ }^{14}$ it is necessary to explain the concepts that underline each method. The PSE model is a kind of deterministic version of Monte Carlo-it simulates the transport process by, in effect, discretizing the transport equation on a grid and then solving the discretized equations. In principle, the limit of the PSE solution for an infinite number of (space, angle, and energy) grid points should be equal to the limit of the Monte Carlo solution for an infinite number of histories. Because the Monte Carlo and PSE methods are based on a direct simulation of the underlying transport equation, they are computationally intensive and costly, compared to other approximate methods that do not directly simulate the transport equation.

The Pencil Beam ${ }^{1}(\mathrm{~PB})$ and Pencil Beam Redefinition ${ }^{14}$ (PBR) methods are such approximate schemes. They are based on the exact analytic solution of a simplified transport equation (the Fermi-Eyges equation) and contain correction factors that attempt to improve their accuracy. Thus, these schemes require the evaluation of analytic expressions rather than the solution of a transport equation. (What transport physics there is, is built into the analytic expressions.) For this reason, the $\mathrm{PB}$ and $\mathrm{PBR}$ schemes are much more computationally efficient than the Monte Carlo or PSE schemes. But, because they are based on a fundamentally less-accurate 
set of equations, they provide fundamentally less-accurate solutions.

The Method of Moments (MM) presented in this paper is a compromise between the computationally intensive Monte Carlo and PSE methods, and the simpler PB and BPR methods. Like the Monte Carlo and PSE methods, the MM scheme uses a transport equation as its underlying description of the physical process. Like the PB schemes, the MM scheme yields an analytic expression, not a large system of discretized equations that must be solved on a grid.

The MM solution is exact only in the following sense: if one were to obtain the PSE solution for an infinite number of grid points or the Monte Carlo solution for an infinite number of histories, and if for each energy one were to calculate the zeroth, first, and second spatial moments of the solution, then these moments would agree exactly with the corresponding spatial moments of the MM solution.

The Method of Moments described in this paper can be generalized in several ways. One can calculate higher-order moments of $\Phi$ and construct more elaborate (and, presumably, more accurate) non-Gaussian representations of the fluence. For example, Lax et al. have represented the dose as the sum of three Gaussians, but the parameters in these Gaussians were derived by Monte Carlo simulations. ${ }^{6}$ Also, Jette's second-order approximation to the Fokker-Planck solution consists of the Fermi-Eyges solution multiplied by a polynomial. ${ }^{11}$ It is possible that by calculating higher moments of $\Phi$ and using the functional forms of Lax or Jette, one can significantly improve upon the estimate of the dose that one can obtain from a simple Gaussian.

One can also apply the Method of Moments to problems containing ionization, bremsstrahlung, and pair production. The extra difficulty is that numerical solutions of Volterra integral equations must be calculated. In addition, one can apply the method to coupled systems of transport equations. For example, the dose deposition due to photon beams is determined by a system of transport equations, one describing the photon fluence, another describing the electron fluence. The calculation of space-angle moments of these equations will apply for that problem, just as it does for the simpler electron problem treated in this paper.

As presented in this paper, the Method of Moments is applicable only in infinite homogeneous media. However, planar inhomogeneities can be treated by a related theory and the Method of Moments can be extended to include largeenergy loss scattering. This work is presently under development.

It is not clear whether these generalizations of the Method of Moments will produce a method that, without empirical corrections, is sufficiently fast and accurate for three-dimensional (3-D) treatment planning. If the resulting scheme contains enough transport physics, depends weakly on empirical corrections, and is computationally efficient, it may suffice for clinical applications. However, such developments must await further study.

\section{ACKNOWLEDGMENTS}

We would like to acknowledge several helpful discussions with Brian Foote, Henk Huizenga, and Jerry Pomraning.

This work was partly supported by NCI Grant No. 5PO1CA59827.

\section{APPENDIX A: THE LINEAR BOLTZMANN, FOKKER-PLANCK, BOLTZMANN-FOKKER-PLANCK, AND BOLTZMANN-CSD EQUATIONS}

Here we describe the linear Boltzmann equation, together with three of its approximations, for a homogeneous medium. All of these equations have been used to model electron transport.

First, the linear Boltzmann (B) equation for electron transport in a homogeneous medium is given by

$$
\boldsymbol{\Omega} \cdot \boldsymbol{\nabla} \Phi(\mathbf{r}, \boldsymbol{\Omega}, E)=L_{\mathrm{B}} \Phi(\mathbf{r}, \boldsymbol{\Omega}, E)+Q(\mathbf{r}, \boldsymbol{\Omega}, E),
$$

where the Boltzmann scattering operator $L_{\mathrm{B}}$ is defined as ${ }^{32,33}$

$$
\begin{aligned}
L_{\mathrm{B}} \Phi(\boldsymbol{\Omega}, E)= & \int_{E}^{\infty} d E^{\prime} \int_{4 \pi} d^{2} \Omega^{\prime} \Sigma_{s}\left(E^{\prime} \rightarrow E, \boldsymbol{\Omega}^{\prime} \cdot \boldsymbol{\Omega}\right) \\
& \times \Phi\left(\boldsymbol{\Omega}^{\prime}, E^{\prime}\right)-\Sigma_{t}(E) \Phi(\boldsymbol{\Omega}, E) .
\end{aligned}
$$

The symbols in these equations are defined earlier in Sec. II, except for $\Sigma_{s}\left(E^{\prime} \rightarrow E, \mu_{0}\right)=$ differential scattering cross section. We have assumed that electrons only lose energy in collisions, so $\Sigma_{s}\left(E^{\prime} \rightarrow E, \mu_{0}\right)=0$ for $E^{\prime}<E$.

Equations (A1) and (A2) provide a very accurate description of electron transport in the absence of electric and magnetic fields. These equations are the underlying mathematical model for Monte Carlo and deterministic simulations of electron transport. In this model, electrons travel in straight lines with no energy loses between collisions. At the collision points, the electrons undergo discrete changes in direction and energy. For electrons, the mean-free path, and the angle and energy change per collision are usually very small.

The differential scattering cross section $\Sigma_{s}$ has the Legendre polynomial expansion

$$
\Sigma_{s}\left(E^{\prime} \rightarrow E, \mu_{0}\right)=\sum_{n=0}^{\infty} \frac{2 n+1}{4 \pi} \Sigma_{s n}\left(E^{\prime} \rightarrow E\right) P_{n}\left(\mu_{0}\right),
$$

where the expansion coefficients $\Sigma_{s n}$ in Eq. (A3) are defined in terms of $\Sigma_{s}$ by

$$
\Sigma_{s n}\left(E^{\prime} \rightarrow E\right)=2 \pi \int_{-1}^{1} d \mu_{0} P_{n}\left(\mu_{0}\right) \Sigma_{s}\left(E^{\prime} \rightarrow E, \mu_{0}\right) .
$$

We also define

$$
\bar{\Sigma}_{s n}(E) \equiv \int_{0}^{E} d E^{\prime} \Sigma_{s n}\left(E \rightarrow E^{\prime}\right), \quad n \geqslant 0 .
$$

For electron transport, one has

$$
\begin{aligned}
& \Sigma_{t}(E)=\bar{\Sigma}_{s 0}(E) \quad \text { (no absorption), } \\
& T(E) \equiv 2\left[\bar{\Sigma}_{s 0}(E)-\bar{\Sigma}_{s 1}(E)\right]=\text { scattering power, }
\end{aligned}
$$




$$
S(E) \equiv \int_{0}^{E} d E^{\prime}\left(E-E^{\prime}\right) \Sigma_{s 0}\left(E \rightarrow E^{\prime}\right)=\text { stopping power. }
$$

Because of the small-angle scattering and small energy loss, the functions $\Sigma_{s n}\left(E^{\prime} \rightarrow E\right)$ are sharply peaked near $E^{\prime}=E$, and the expansion in Eq. (A3) requires a large number of terms to achieve acceptable accuracy.

The Fokker-Planck (FP) approximation to Eq. (A1) is

$$
\boldsymbol{\Omega} \cdot \boldsymbol{\nabla} \Phi(\mathbf{r}, \boldsymbol{\Omega}, E)=L_{\mathrm{FP}} \Phi(\mathbf{r}, \boldsymbol{\Omega}, E)+Q(\mathbf{r}, \boldsymbol{\Omega}, E),
$$

where the Fokker-Planck scattering operator $L_{\mathrm{FP}}$ is defined as $^{32,33}$

$$
\begin{aligned}
L_{\mathrm{FP}} \Phi(\boldsymbol{\Omega}, E) \equiv & \frac{T(E)}{4}\left[\frac{\partial}{\partial \mu}\left(1-\mu^{2}\right) \frac{\partial}{\partial \mu}\right. \\
& \left.+\frac{1}{1-\mu^{2}} \frac{\partial^{2}}{\partial \phi^{2}}\right] \Phi(\boldsymbol{\Omega}, E) \\
& +\frac{\partial}{\partial E} S(E) \Phi(\boldsymbol{\Omega}, E) .
\end{aligned}
$$

A classic derivation of the FP equation is given by Chandrasekhar. ${ }^{32}$ Also, Pomraning ${ }^{33}$ has shown that Eqs. (A9) and (A10) can be derived from Eqs. (A1) and (A2) in an asymptotic limit in which large-angle scattering and large energy-loss scattering are negligible. Here $L_{\mathrm{FP}}$ is a much simpler operator than $L_{\mathrm{B}}$, but Chandrasekhar's and Pomraning's analyses both show that $L_{\mathrm{FP}}$ omits large-angle and large energy-loss scattering. Equation (A10) describes a scattering process in which electrons simultaneously lose energy and change their direction of flight continuously as functions of pathlength. is

The Boltzmann-CSD (BCSD) approximation to Eq. (A1)

$$
\boldsymbol{\Omega} \cdot \boldsymbol{\nabla} \Phi(\mathbf{r}, \boldsymbol{\Omega}, E)=L_{\mathrm{BCSD}} \Phi(\mathbf{r}, \boldsymbol{\Omega}, E)+Q(\mathbf{r}, \boldsymbol{\Omega}, E),
$$

where $L_{\mathrm{BCSD}}$ is defined as

$$
\begin{aligned}
L_{\mathrm{BCSD}} \Phi(\boldsymbol{\Omega}, E)= & \int_{4 \pi} d \boldsymbol{\Omega}^{\prime} \Sigma_{s}\left(E, \boldsymbol{\Omega} \cdot \boldsymbol{\Omega}^{\prime}\right) \Phi\left(\boldsymbol{\Omega}^{\prime}, E\right) \\
& -\Sigma_{t}(E) \Phi(\boldsymbol{\Omega}, E)+\frac{\partial}{\partial E} S(E) \Phi(\boldsymbol{\Omega}, E),
\end{aligned}
$$

with

$$
\Sigma_{s}\left(E, \mu_{0}\right) \equiv \int_{0}^{E} d E^{\prime} \Sigma_{s}\left(E \rightarrow E^{\prime}, \mu_{0}\right) .
$$

In the BCSD model, electrons travel in straight lines between scattering events, at which their direction of flight changes discretely. This aspect of the model is physically correct. However, electrons lose energy continuously as a function of pathlength, and this is approximate. The advantage of the BCSD model over the FP model is that it permits large-angle scattering.

The Boltzmann-Fokker-Planck (BFP) approximation to Eq. (A1) is

$$
\boldsymbol{\Omega} \cdot \boldsymbol{\nabla} \Phi(\mathbf{r}, \boldsymbol{\Omega}, E)=L_{\mathrm{BFP}} \Phi(\mathbf{r}, \boldsymbol{\Omega}, E)+Q(\mathbf{r}, \boldsymbol{\Omega}, E),
$$

where $L_{\mathrm{BFP}}$, the BFP scattering operator, contains both Boltzmann-like and Fokker-Planck-like scattering terms. ${ }^{34,35}$

$$
\begin{aligned}
L_{\mathrm{BFP}} \Phi(\boldsymbol{\Omega}, E)= & \int_{E^{\prime}=0}^{\infty} d E^{\prime} \int_{4 \pi} d^{2} \Omega^{\prime} \sigma_{s}\left(E^{\prime} \rightarrow E, \boldsymbol{\Omega}^{\prime} \cdot \boldsymbol{\Omega}\right) \\
& \times \Phi\left(\boldsymbol{\Omega}^{\prime}, E^{\prime}\right)-\sigma_{t}(E) \Phi(\boldsymbol{\Omega}, E)+\frac{t(E)}{4} \\
& \times\left(\frac{\partial}{\partial \mu}\left(1-\mu^{2}\right) \frac{\partial}{\partial \mu}+\frac{1}{1-\mu^{2}} \frac{\partial^{2}}{\partial \phi^{2}}\right) \\
& \times \Phi(\boldsymbol{\Omega}, E)+\frac{\partial}{\partial E} s(E) \Phi(\boldsymbol{\Omega}, E) .
\end{aligned}
$$

In practical applications, the decomposition of $\Sigma_{s}$ and $\Sigma_{t}$ into $\sigma_{s}, \sigma_{t}, t$, and $s$ is nonunique, but the concept is for the Boltzmann-like terms $\left(\sigma_{s}\right.$ and $\left.\sigma_{t}\right)$ to describe large-angle and large energy-loss scattering, and the Fokker-Planck-like terms $(t$ and $s)$ to describe small-angle and small energy-loss scattering. The advantage of this description is that it makes the kernel $\sigma_{s}\left(E^{\prime} \rightarrow E, \mu_{0}\right)$ a less sharply peaked function of $E$ and $\mu_{0}$ than $\Sigma_{s}\left(E^{\prime} \rightarrow E, \mu_{0}\right)$, and hence easier to simulate numerically.

In Appendix B, we develop the Method of Moments for the BFP scattering operator because it includes the $B$, FP, and BCSD scattering operators as special cases. [If one sets $t=s=0, \sigma_{s}=\Sigma_{s}$, and $\sigma_{t}=\Sigma_{t}$, then Eq. (A15) reduces to Eq. (A2). If one sets $\sigma_{s}=\sigma_{t}=0, t=T$, and $s=S$, then Eq. (A15) reduces to Eq. (A10). If one sets $t=0, s=S, \sigma_{t}=\Sigma_{t}$, and $\sigma_{s}\left(E^{\prime} \rightarrow E, \mu_{0}\right)=\delta\left(E^{\prime}-E\right) \Sigma_{s}\left(E^{\prime}, \mu_{0}\right)$, then Eq. (A15) reduces to Eq. (A12).]

We make the standard assumptions that $\sigma_{s}$ and $\sigma_{t}$ are related by the analogs of Eqs. (A3)-(A6), i.e.,

$$
\begin{aligned}
& \sigma_{s}\left(E^{\prime} \rightarrow E, \mu_{0}\right)=\sum_{n=0}^{\infty} \frac{2 n+1}{4 \pi} \sigma_{s n}\left(E^{\prime} \rightarrow E\right) P_{n}\left(\mu_{0}\right), \\
& \sigma_{s n}\left(E^{\prime} \rightarrow E\right)=2 \pi \int_{0}^{\infty} d \mu_{0} P_{n}\left(\mu_{0}\right) \sigma_{s}\left(E^{\prime} \rightarrow E, \mu_{0}\right),
\end{aligned}
$$

$$
\begin{aligned}
& \sigma_{s n}(E) \equiv \int_{0}^{E} d E^{\prime} \sigma_{s n}\left(E \rightarrow E^{\prime}\right), \quad n \geqslant 0, \\
& \sigma_{t}(E)=\sigma_{s 0}(E) .
\end{aligned}
$$

Finally, we make use of special properties of the spherical harmonic functions $Y_{n, m}(\boldsymbol{\Omega})$. These functions are defined by ${ }^{36,37}$

$$
Y_{n, m}(\boldsymbol{\Omega})=\alpha_{n, m} P_{n,|m|}(\mu) e^{i m \phi}, \quad 0 \leqslant|m| \leqslant n,
$$

where $P_{n, m}(\mu)$ are the associated Legendre functions, and

$$
\alpha_{n, m}=\left(\frac{2 n+1}{4 \pi} \frac{(n-|m|) !}{(n+|m|) !}\right)^{1 / 2} \text {. }
$$


Here $Y_{n, m}$ satisfy the orthogonality condition

$$
\int_{4 \pi} d^{2} \Omega Y_{n, m}^{*}(\mathbf{\Omega}) Y_{p, q}(\boldsymbol{\Omega})=\delta_{n, p} \delta_{m, q},
$$

the differential equation

$$
\begin{gathered}
\left(\frac{\partial}{\partial \mu}\left(1-\mu^{2}\right) \frac{\partial}{\partial \mu}+\frac{1}{1-\mu^{2}} \frac{\partial^{2}}{\partial \phi^{2}}\right) Y_{n, m}(\mathbf{\Omega}) \\
=-n(n+1) Y_{n, m}(\mathbf{\Omega}),
\end{gathered}
$$

and the addition theorem,

$$
P_{k}\left(\boldsymbol{\Omega} \cdot \boldsymbol{\Omega}^{\prime}\right)=\frac{4 \pi}{2 k+1} \sum_{j=-k}^{k} Y_{k, j}^{*}\left(\boldsymbol{\Omega}^{\prime}\right) Y_{k, j}(\boldsymbol{\Omega}),
$$

where $P_{k}(\mu)$ is the $k$ th Legendre polynomial. Also $^{37}$ (see the Introduction for the definition of $\Omega_{i}$ ) note the following.

(i) The function $f(\boldsymbol{\Omega})=1$ is proportional to $Y_{0,0}(\boldsymbol{\Omega})$. (ii) For $1 \leqslant i \leqslant 3$, the functions $f_{i}(\boldsymbol{\Omega})=\Omega_{i}$ are linear combinations of spherical harmonic functions of order unity $\left(Y_{1, m}\right.$ for $\left.-1 \leqslant m \leqslant 1\right)$.

(iii) For $1 \leqslant i, j \leqslant 3$, the functions $f_{i j}(\mathbf{\Omega})=3 \Omega_{i} \Omega_{j}-\delta_{i j}$ are linear combinations of spherical harmonic functions of order two $\left(Y_{2, m}\right.$ for $\left.-2 \leqslant m \leqslant 2\right)$.

(A27)

\section{APPENDIX B: CALCULATION OF THE SPACE-ANGLE MOMENTS OF THE FLUENCE}

To derive equations for the space-angle moments of $\Phi$, we first multiply the BFP equation (A14) by an arbitrary spherical harmonic function $Y_{n, m}(\boldsymbol{\Omega})$ and integrate over $\boldsymbol{\Omega}$. We obtain

$$
\begin{aligned}
& \int_{4 \pi} d^{2} \boldsymbol{\Omega} Y_{n, m}(\boldsymbol{\Omega}) \boldsymbol{\Omega} \cdot \boldsymbol{\nabla} \Phi(\mathbf{r}, \boldsymbol{\Omega}, E)=\int_{E}^{\infty} d E^{\prime} \int_{4 \pi} d^{2} \Omega\left[\int_{4 \pi} d^{2} \Omega^{\prime} Y_{n, m}\left(\boldsymbol{\Omega}^{\prime}\right) \sigma_{s}\left(E^{\prime} \rightarrow E, \boldsymbol{\Omega}^{\prime} \cdot \boldsymbol{\Omega}\right)\right] \Phi\left(\mathbf{r}, \boldsymbol{\Omega}, E^{\prime}\right)-\sigma_{t}(E) \\
& \times\left(\int_{4 \pi} d^{2} \Omega Y_{n, m}(\boldsymbol{\Omega}) \Phi(\mathbf{r}, \boldsymbol{\Omega}, E)\right)+\frac{t(E)}{4} \int_{4 \pi} d^{2} \Omega \Phi(\mathbf{r}, \boldsymbol{\Omega}, E)\left(\frac{\partial}{\partial \mu}\left(1-\mu^{2}\right) \frac{\partial}{\partial \mu}\right. \\
& \left.+\frac{1}{1-\mu^{2}} \frac{\partial^{2}}{\partial \phi^{2}}\right) Y_{n, m}(\mathbf{\Omega})+\frac{\partial}{\partial E} s(E)\left(\int_{4 \pi} d^{2} \Omega Y_{n, m}(\mathbf{\Omega}) \Phi(\mathbf{r}, \mathbf{\Omega}, E)\right) \\
& +\left(\int_{4 \pi} d^{2} \Omega Y_{n, m}(\mathbf{\Omega}) Q(\mathbf{r}, \mathbf{\Omega}, E)\right) .
\end{aligned}
$$

In the first term on the right we have interchanged integrations over $\boldsymbol{\Omega}$ and $\boldsymbol{\Omega}^{\prime}$ and have then replaced $\boldsymbol{\Omega}^{\prime}$ by $\boldsymbol{\Omega}$ and vice versa. In the third term on the right, we have integrated by parts.

Equations (A16), (A22), and (A24) now imply

$$
\begin{aligned}
\int_{4 \pi} d^{2} \Omega^{\prime} Y_{n, m}\left(\mathbf{\Omega}^{\prime}\right) \sigma_{s}\left(E^{\prime} \rightarrow E, \mathbf{\Omega}^{\prime} \cdot \mathbf{\Omega}\right) \\
=\sum_{k=0}^{\infty} \frac{2 k+1}{4 \pi} \sigma_{s k}\left(E^{\prime} \rightarrow E\right) \int_{4 \pi} d^{2} \Omega^{\prime} \\
\quad \times Y_{n, m}\left(\mathbf{\Omega}^{\prime}\right) P_{k}\left(\mathbf{\Omega} \cdot \mathbf{\Omega}^{\prime}\right) \\
=\sum_{k=0}^{\infty} \frac{2 k+1}{4 \pi} \sigma_{s k}\left(E^{\prime} \rightarrow E\right)\left(\frac{4 \pi}{2 k+1} Y_{k, m}(\mathbf{\Omega}) \delta_{k n}\right) \\
=\sigma_{s n}\left(E^{\prime} \rightarrow E\right) Y_{n, m}(\mathbf{\Omega}) .
\end{aligned}
$$

$$
\begin{aligned}
\int_{4 \pi} d^{2} \Omega Y_{n, m}(\boldsymbol{\Omega}) \boldsymbol{\Omega} \cdot \boldsymbol{\nabla} \Phi(\mathbf{r}, \boldsymbol{\Omega}, E) \\
\quad+L_{n}\left(\int_{4 \pi} d^{2} \Omega Y_{n, m}(\boldsymbol{\Omega}) \Phi(\mathbf{r}, \boldsymbol{\Omega}, E)\right) \\
=\left(\int_{4 \pi} d^{2} \Omega Y_{n, m}(\mathbf{\Omega}) Q(\mathbf{r}, \boldsymbol{\Omega}, E)\right) .
\end{aligned}
$$

Here $L_{n}$ are reduced scattering operators, acting only on $E$, defined by

$$
\begin{aligned}
L_{n} \Phi(E) \equiv & -\frac{\partial}{\partial E} s(E) \Phi(E)+\left(\sigma_{t}(E)+\frac{t(E)}{4} n(n+1)\right) \\
& \times \Phi(E)-\int_{E}^{\infty} d E^{\prime} \sigma_{s n}\left(E^{\prime} \rightarrow E\right) \Phi\left(E^{\prime}\right) .
\end{aligned}
$$


equation $L_{n} f(E)=g(E)$ exists] under very mild conditions on $s, \sigma_{t}, t$, and $\sigma_{s n}$ that are satisfied in practical applications.

Equations (B3) and (A25) now imply

$$
\begin{gathered}
\int_{4 \pi} d^{2} \Omega \boldsymbol{\Omega} \cdot \nabla \Phi(\mathbf{r}, \boldsymbol{\Omega}, E)+L_{0}\left(\int_{4 \pi} d^{2} \Omega \Phi(\mathbf{r}, \boldsymbol{\Omega}, E)\right) \\
=\left(\int_{4 \pi} d^{2} \Omega Q(\mathbf{r}, \boldsymbol{\Omega}, E)\right) .
\end{gathered}
$$

For $1 \leqslant i \leqslant 3$, Eqs. (B3) and (A26) imply

$$
\begin{aligned}
\int_{4 \pi} d^{2} \Omega & \Omega_{i} \boldsymbol{\Omega} \cdot \nabla \Phi(\mathbf{r}, \boldsymbol{\Omega}, E) \\
& +L_{1}\left(\int_{4 \pi} d^{2} \Omega \Omega_{i} \Phi(\mathbf{r}, \boldsymbol{\Omega}, E)\right) \\
= & \left(\int_{4 \pi} d^{2} \Omega \Omega_{i} Q(\mathbf{r}, \boldsymbol{\Omega}, E)\right) .
\end{aligned}
$$

Also, for $1 \leqslant i, j \leqslant 3$, Eqs. (B3) and (A27) imply

$$
\begin{aligned}
\int_{4 \pi} d^{2} \Omega\left(3 \Omega_{i} \Omega_{j}-\delta_{i j}\right) \boldsymbol{\Omega} \cdot \boldsymbol{\nabla} \Phi(\mathbf{r}, \boldsymbol{\Omega}, E) \\
\quad+L_{2}\left(\int_{4 \pi} d^{2} \Omega\left(3 \Omega_{i} \Omega_{j}-\delta_{i j}\right) \Phi(\mathbf{r}, \boldsymbol{\Omega}, E)\right) \\
=\left(\int_{4 \pi} d^{2} \Omega\left(3 \Omega_{i} \Omega_{j}-\delta_{i j}\right) Q(\mathbf{r}, \boldsymbol{\Omega}, E)\right) .
\end{aligned}
$$

Equations (B5)-(B7) are exact, derived without approximation from Eq. (B1). Higher-order equations can also be derived, but we will not do this here.

Next, we recall the space-angle integration operator $\langle\cdot\rangle$ defined by Eq. (6). Integrating Eqs. (B5)-(B7) over $\mathbf{r}$ and assuming that for each $E>0, \Phi \rightarrow 0$ as $|\mathbf{r}| \rightarrow \infty$, we obtain

$$
\begin{aligned}
& L_{0}\langle\Phi\rangle(E)=\langle Q\rangle(E), \\
& L_{1}\left\langle\Omega_{i} \Phi\right\rangle(E)=\left\langle\Omega_{i} Q\right\rangle(E), \\
& L_{2}\left\langle\left(3 \Omega_{i} \Omega_{j}-\delta_{i j}\right) \Phi\right\rangle(E)=\left\langle\left(3 \Omega_{i} \Omega_{j}-\delta_{i j}\right) Q\right\rangle(E) .
\end{aligned}
$$

These are exact infinite-medium equations for the angular moments of $\Phi$ up to order 2. In the CSD approximation, they reduce to first-order ode's that can be solved explicitly. (These ode's, together with the ode's for the higher angular moments, yield the components of the Goudsmit-Saunderson distribution. ${ }^{38}$ ) If the CSD approximation is not valid, Eqs. (B8)-(B10) must generally be solved numerically, but the structure of these equations admits a simple computational algorithm.

Next, we multiply Eq. (B5) by $x_{i}$ and Eq. (B6) by $x_{j}$ (see the Introduction for the definition of $x_{i}$ ) and integrate over $\mathbf{r}$ to obtain

$\left\langle x_{i} \boldsymbol{\Omega} \cdot \boldsymbol{\nabla} \Phi\right\rangle(E)+L_{0}\left\langle x_{i} \Phi\right\rangle(E)=\left\langle x_{i} Q\right\rangle(E), \quad 1 \leqslant i \leqslant 3$,

$\left\langle x_{j} \Omega_{i} \boldsymbol{\Omega} \cdot \nabla \Phi\right\rangle(E)+L_{1}\left\langle x_{j} \Omega_{i} \Phi\right\rangle(E)=\left\langle x_{j} \Omega_{i} Q\right\rangle(E)$,

$1 \leqslant i, j \leqslant 3$.
Integration by parts over $\mathbf{r}$ yields

$$
\begin{aligned}
\left\langle x_{i} \boldsymbol{\Omega} \cdot \boldsymbol{\nabla} \Phi\right\rangle(E)= & -\left\langle\left(\boldsymbol{\Omega} \cdot \boldsymbol{\nabla} x_{i}\right) \Phi\right\rangle(E)=-\left\langle\Omega_{i} \Phi\right\rangle(E), \\
\left\langle x_{j} \Omega_{i} \boldsymbol{\Omega} \cdot \boldsymbol{\nabla} \Phi\right\rangle(E) & =-\left\langle\left(\boldsymbol{\Omega} \cdot \boldsymbol{\nabla} x_{j} \Omega_{i}\right) \Phi\right\rangle(E) \\
& =-\left\langle\Omega_{j} \Omega_{i} \Phi\right\rangle(E),
\end{aligned}
$$

so Eqs. (B11) and (B12) may be written as

$L_{0}\left\langle x_{i} \Phi\right\rangle(E)=\left\langle x_{i} Q\right\rangle(E)+\left\langle\Omega_{i} \Phi\right\rangle(E), \quad 1 \leqslant i \leqslant 3$,

$L_{1}\left\langle x_{j} \Omega_{i} \Phi\right\rangle(E)=\left\langle x_{j} \Omega_{i} Q\right\rangle(E)+\left\langle\Omega_{j} \Omega_{i} \Phi\right\rangle(E), \quad 1 \leqslant i, j \leqslant 3$.

Having previously determined $\left\langle\Omega_{i} \Phi\right\rangle$ and $\left\langle\Omega_{i} \Omega_{j} \Phi\right\rangle$, we can solve these equations for $\left\langle x_{i} \Phi\right\rangle$ and $\left\langle x_{j} \Omega_{i} \Phi\right\rangle$.

Finally, we multiply Eq. (B5) by $x_{i} x_{j}$ and integrate over $\mathbf{r}$ to obtain

$$
\left\langle x_{i} x_{j} \boldsymbol{\Omega} \cdot \boldsymbol{\nabla} \Phi\right\rangle(E)+L_{0}\left\langle x_{i} x_{j} \Phi\right\rangle(E)=\left\langle x_{i} x_{j} Q\right\rangle(E) .
$$

Integration by parts yields

$$
\begin{aligned}
\left\langle x_{i} x_{j} \boldsymbol{\Omega} \cdot \boldsymbol{\nabla} \Phi\right\rangle(E) & =-\left\langle\left(\boldsymbol{\Omega} \cdot \boldsymbol{\nabla} x_{i} x_{j}\right) \Phi\right\rangle(E) \\
& =-\left\langle\left(\Omega_{i} x_{j}+\Omega_{j} x_{i}\right) \Phi\right\rangle(E),
\end{aligned}
$$

so Eq. (B17) may be written as

$$
\begin{aligned}
& L_{0}\left\langle x_{i} x_{j} \Phi\right\rangle(E)=\left\langle x_{i} x_{j} Q\right\rangle(E)+\left\langle\Omega_{i} x_{j} \Phi\right\rangle(E)+\left\langle\Omega_{j} x_{i} \Phi\right\rangle(E), \\
& \quad 1 \leqslant i, j \leqslant 3 .
\end{aligned}
$$

Having previously determined the space-angle moments $\left\langle\Omega_{i} x_{j} \Phi\right\rangle$ for all $i$ and $j$, we can solve these equations for the second-order spatial moments $\left\langle x_{i} x_{j} \Phi\right\rangle(E)$.

To summarize, we note the following.

(1) The zeroth-order spatial moment $\langle\Phi\rangle(E)$ is determined by solving Eq. (B8).

(2) The first-order spatial moments $\left\langle x_{i} \Phi\right\rangle(E)$ are determined by first solving Eqs. (B9) for $\left\langle\Omega_{i} \Phi\right\rangle(E)$, and then solving Eqs. (B15).

(3) The second-order spatial moments $\left\langle x_{i} x_{j} \Phi\right\rangle(E)$ are determined by first solving Eqs. (B10) for $\left\langle\Omega_{i} \Omega_{j} \Phi\right\rangle(E)$, then solving Eqs. (B16) for $\left\langle x_{j} \Omega_{i} \Phi\right\rangle(E)$, and then solving Eqs. (B19).

(In all cases, we assume the "initial" condition $\lim _{E \rightarrow \infty} \Phi$ $=0$.)

We emphasize that these results are exact and fundamental: they apply to any transport equation of the form described by Eqs. (A14) and (A15).

Some observations are made:

(i) To calculate the zeroth space-angle moment of $\Phi$, one only needs to know $\sigma_{t}(E)$ and $\sigma_{s 0}\left(E^{\prime} \rightarrow E\right)$.

(ii) To calculate the first space-angle moments of $\Phi$, one also needs to know $\sigma_{s 1}\left(E^{\prime} \rightarrow E\right)$.

(iii) To calculate the second space-angle moments of $\Phi$, one also needs to know $\sigma_{s 2}\left(E^{\prime} \rightarrow E\right)$.

Therefore, the implementation of the Method of Moments requires knowledge of the zeroth, first, and second Legendre moments of the differential scattering cross section. The Fokker-Planck approximation is based only on knowledge of the zeroth and first Legendre moments, through the stopping power and the scattering power. The extra information 
required by the Method of Moments corresponds to the degree and importance of large-angle and large energy-loss scattering in the problem.

Another aspect of the Method of Moments is that one must solve a system of equations, each of which is of the form

$$
L_{n} f(E)=g(E),
$$

where $L_{n}$ is defined by Eq. (B4). For the exact Linear Boltzmann equation, $s(E)=t(E)=0$, and Eq. (B20) reduces to a simple Volterra integral equation. ${ }^{39}$ In general, Eq. (B20) must be solved numerically. However, because electrons "flow" from larger to smaller energies, it is possible to discretize Eq. (B20) on an energy grid and solve the resulting discrete equations sequentially (one first solves for the largest value of $E$, then the next largest value, and so on). For problems with CSD, Eq. (B20) reduces to a simple first-order ordinary differential equation that can be solved analytically. We consider this case next.

\section{APPENDIX C: EXPLICIT RESULTS FOR THE BOLTZMANN-CSD EQUATION}

We now specialize our results to the case of the Boltzmann-CSD equation, defined by Eqs. (A11) and (A12). This is the most physically realistic equation for which $\langle\Phi\rangle(E)$, $\left\langle x_{i} \Phi\right\rangle(E)$, and $\left\langle x_{i} x_{j} \Phi\right\rangle(E)$ can be obtained explicitly. We consider a point source at $\mathbf{r}=0$, emitting electrons at energy $E_{0}$ in directions determined by an azimuthally symmetric probability distribution function $p(\mu)$ :

$$
Q(\mathbf{r}, \mathbf{\Omega}, E)=Q_{0} \delta(\mathbf{r}) \frac{p(\mu)}{2 \pi} \delta\left(E-E_{0}\right) .
$$

Here

$$
\int_{-1}^{1} d \mu p(\mu)=1
$$

[There are at least two types of functions $p$ that are relevant in medical physics: (i) $p(\mu)=\delta(\mu-1)$ corresponds to a monodirectional beam, and (ii) in the Compton scattering of photons off electrons, electrons are released according to the Compton scattering pdf $p(\mu)$. Other choices of $p(\mu)$ occur for beams that are not perfectly monodirectional.]

The relevant BCSD transport equation is

$$
\begin{aligned}
\boldsymbol{\Omega} \cdot \boldsymbol{\nabla} & \Phi(\mathbf{r}, \boldsymbol{\Omega}, E)+\Sigma_{t}(E) \Phi(\mathbf{r}, \boldsymbol{\Omega}, E) \\
& =\int_{4 \pi} d \Omega^{\prime} \Sigma_{s}\left(E, \boldsymbol{\Omega} \cdot \boldsymbol{\Omega}^{\prime}\right) \Phi\left(\mathbf{r}, \boldsymbol{\Omega}^{\prime}, E\right) \\
& +\frac{\partial}{\partial E} S(E) \Phi(\mathbf{r}, \boldsymbol{\Omega}, E)+Q_{0} \delta(\mathbf{r}) \frac{p(\mu)}{2 \pi} \delta\left(E-E_{0}\right) .
\end{aligned}
$$

This is of the form of Eqs. (A14) and (A15) if we define $\sigma_{t}(E)=\Sigma_{t}(E), \sigma_{s}\left(E^{\prime} \rightarrow E, \mu_{0}\right)=\delta\left(E^{\prime}-E\right) \sigma_{s}\left(E, \mu_{0}\right), t(E)$ $=0$, and $s(E)=S(E)$, with $\Sigma_{s}\left(E, \mu_{0}\right)$ defined by Eq. (A13). Equations (A16) and (A18) imply

$$
\begin{aligned}
& \sigma_{s}\left(E^{\prime} \rightarrow E, \mu_{0}\right)=\sum_{n=0}^{\infty} \frac{2 n+1}{4 \pi} \delta\left(E^{\prime}-E\right) \Sigma_{s n}(E) P_{n}\left(\mu_{0}\right), \\
& \sigma_{s n}\left(E^{\prime} \rightarrow E\right)=\delta\left(E^{\prime}-E\right) \Sigma_{s n}(E), \\
& \Sigma_{s n}(E)=2 \pi \int_{-1}^{1} d \mu_{0} P_{n}\left(\mu_{0}\right) \Sigma_{s}\left(E, \mu_{0}\right) .
\end{aligned}
$$

Thus, Eq. (B4) reduces to

$$
L_{n} \Phi(E)=-\frac{\partial}{\partial E} S(E) \Phi(E)+\Sigma_{a n}(E) \Phi(E), \quad n \geqslant 0,
$$

where $\Sigma_{a n}(E)$ is defined by Eq. (7).

Now let us calculate the equation for the zeroth order space-angle moment $\langle\Phi\rangle(E)$. Equations (B8) and (C7) yield the following equation for $\langle\Phi\rangle(E)$ :

$$
-\frac{\partial}{\partial E} S(E)\langle\Phi\rangle(E)=Q_{0} \delta\left(E-E_{0}\right) .
$$

Next, we calculate the equations for the first-order spaceangle moments of $\Phi$. Equations (B9), (C7), (C1), and (C2) yield

$$
\begin{gathered}
-\frac{\partial}{\partial E} S(E)\left\langle\Omega_{i} \Phi\right\rangle(E)+\frac{T(E)}{2}\left\langle\Omega_{i} \Phi\right\rangle(E) \\
=\hat{\mu} Q_{0} \delta_{i 3} \delta\left(E-E_{0}\right),
\end{gathered}
$$

where

$$
\widehat{\mu^{n}} \equiv \int_{-1}^{1} d \mu \mu^{n} p(\mu), \quad n=1,2 .
$$

Equations (B15) and (C7) now yield

$$
-\frac{\partial}{\partial E} S(E)\left\langle x_{i} \Phi\right\rangle(E)=\left\langle\Omega_{i} \Phi\right\rangle(E) .
$$

Finally, we calculate equations for the second-order space-angle moments of $\Phi$. Equations (B10), (C1), and (C7) yield

$$
\begin{aligned}
- & \frac{\partial}{\partial E} S(E)\left\langle\left(3 \Omega_{i} \Omega_{j}-\delta_{i j}\right) \Phi\right\rangle(E) \\
& +\Sigma_{a 2}(E)\left\langle\left(3 \Omega_{i} \Omega_{j}-\delta_{i j}\right) \Phi\right\rangle(E) \\
& =\left(-\delta_{i j}+3 \delta_{i 3} \delta_{j 3}\right)\left(\frac{3 \mu^{2}-1}{2}\right) Q_{0} \delta\left(E-E_{0}\right) .
\end{aligned}
$$

Next, Eqs. (B16), (C7), and (A7) yield

$$
\begin{aligned}
& -\frac{\partial}{\partial E} S(E)\left\langle x_{i} \Omega_{j} \Phi\right\rangle(E)+\frac{T(E)}{2}\left\langle x_{i} \Omega_{j} \Phi\right\rangle(E) \\
& \quad=\left\langle\Omega_{i} \Omega_{j} \Phi\right\rangle(E) .
\end{aligned}
$$

Finally, Eqs. (B19) and (C7) yield

$$
-\frac{\partial}{\partial E} S(E)\left\langle x_{i} x_{j} \Phi\right\rangle(E)=2\left\langle x_{i} \Omega_{j} \Phi\right\rangle(E) .
$$

For the special case of a monodirectional beam source, for 
which $p(\mu)=\delta(\mu-1)$ and $\hat{\mu}=\widehat{\mu^{2}}=1$, we have written the explicit solutions of Eqs. (C8)-(C14) in Eqs. 8-15.

Equations (C8)-(C14) show that to determine the MMBSCD solution, it is necessary to know the stopping power $S(E)$, the scattering power $T(E)$, and $\Sigma_{a 2}(E)$. For problems with negligible large-angle scattering, Eq. (D16) shows that $\Sigma_{a 2}(E)=1.5 T(E)$, and hence it is only necessary to know the stopping and scattering powers. (This is the physical regime in which Fokker-Planck theory is valid.) However, for problems with significant large-angle scattering, $\Sigma_{a 2}(E)$ $<1.5 T(E)$; here Fokker-Planck theory is not valid, and one must also know $\Sigma_{a 2}(E)$.

The BCSD and FP equations have the same value of $\Sigma_{a 1}(E)$, but different values of $\Sigma_{a 2}(E)$. Therefore, the FP solution has the same zeroth and first-order space-angle moments of $\Phi$ as the BCSD solution, but different second-order space-angle moments. This implies that for each $E$, the mean electron displacements are the same for the BCSD and FP equations, but the mean-squared displacements are different.

\section{APPENDIX D: RELATIONSHIP OF THE MM-BCSD SOLUTION TO THE FERMI-EYGES AND FERMI-AGE SOLUTIONS}

Here we shall discuss the relationship between the Method of Moments solution developed in this paper and the earlier Fermi-Eyges ${ }^{4,5}$ and Fermi-Age ${ }^{26}$ solutions.

To do this, we observe that the MM scalar fluence $F(\mathbf{r}, E)$, defined by Eq. (21), is the exact solution of the following anisotropic drift-diffusion problem:

$$
\begin{aligned}
-\frac{\partial}{\partial E} S(E) F(\mathbf{r}, E)= & \mathscr{D}_{r}(E)\left(\frac{\partial^{2} F}{\partial x^{2}}(\mathbf{r}, E)+\frac{\partial^{2} F}{\partial y^{2}}(\mathbf{r}, E)\right) \\
& +\mathscr{D}_{z}(E) \frac{\partial^{2} F}{\partial z^{2}}(\mathbf{r}, E) \\
& -v(E) \frac{\partial F}{\partial z}(\mathbf{r}, E), \\
& 0<E<E_{0}, \quad-\infty<x, y, z<\infty, \\
F\left(\mathbf{r}, E_{0}\right)=Q_{0} \delta(x) \delta(y) \delta(z), & \text { as }|\mathbf{r}| \rightarrow \infty, \text { for all } 0<E<E_{0},
\end{aligned}
$$

where

$$
\begin{aligned}
& \mathscr{D}_{r}(E) \equiv \frac{\left\langle x_{1} \Omega_{1} \Phi\right\rangle(E)}{\langle\Phi\rangle(E)}=\frac{\left\langle x_{2} \Omega_{2} \Phi\right\rangle(E)}{\langle\Phi\rangle(E)}, \\
& \mathscr{D}_{z}(E) \equiv \frac{\left\langle\left[x_{3}-\bar{x}_{3}(E)\right] \Omega_{3} \Phi\right\rangle(E)}{\langle\Phi\rangle(E)}, \quad \bar{x}_{3}(E)=\frac{\left\langle x_{3} \Phi\right\rangle(E)}{\langle\Phi\rangle(E)}, \\
& v(E) \equiv \frac{\left\langle\Omega_{3} \Phi\right\rangle(E)}{\langle\Phi\rangle(E)} .
\end{aligned}
$$

[One can show that $F$ satisfies these equations either by introducing $F$ into them and directly verifying that they are satisfied, or by solving Eqs. (D1)-(D3) using Fourier transforms in $x, y$, and $z$. We will not present the details here.]
Introducing Eqs. (6)-(15) into Eqs. (D4)-(D6), we obtain explicitly

$$
\begin{aligned}
\mathscr{D}_{r}(E)= & \frac{e^{-f_{1}(E)}}{3} \int_{E}^{E_{0}} \frac{d E^{\prime}}{S\left(E^{\prime}\right)}\left(1-e^{-f_{2}\left(E^{\prime}\right)}\right) e^{f_{1}\left(E^{\prime}\right)} \\
\mathscr{D}_{z}(E)= & e^{-f_{1}(E)} \int_{E}^{E_{0}} \frac{d E^{\prime}}{S\left(E^{\prime}\right)}\left[\frac{1}{3}\left(1+2 e^{-f_{2}\left(E^{\prime}\right)}\right) e^{f_{1}\left(E^{\prime}\right)}\right. \\
& \left.-e^{-f_{1}\left(E^{\prime}\right)}\right] \\
v(E)= & e^{-f_{1}(E)}
\end{aligned}
$$

The function $f_{1}(E)$ [Eq. (7)] has a simple physical interpretation, which we will now describe. Using

$$
\begin{aligned}
S\left(E^{\prime}\right)= & -\frac{d E^{\prime}}{d s} \\
= & \text { the rate of energy loss of an electron at } \\
& \text { energy } E \text { per unit pathlength, }
\end{aligned}
$$

and

$$
\begin{aligned}
T\left(E^{\prime}\right)= & 2 \Sigma_{a 1}\left(E^{\prime}\right)=\frac{d\left\langle\theta^{2}\right\rangle}{d s} \\
= & \text { the mean-squared deflection of an electron at } \\
& \text { energy } E^{\prime} \text { per unit pathlength, }
\end{aligned}
$$

one obtains

$$
\begin{aligned}
2 \frac{\Sigma_{a 1}\left(E^{\prime}\right)}{S\left(E^{\prime}\right)} d E^{\prime}= & d\left\langle\theta^{2}\right\rangle\left(E^{\prime}\right) \\
= & \text { the mean-squared angular deflection } \\
& \text { experienced by an electron while }
\end{aligned}
$$$$
\text { slowing down from } E^{\prime}+d E^{\prime} \text { to } E^{\prime} \text {. }
$$

Therefore, $f_{1}(E)$ is one-half the integral of the infinitesimal mean-squared angular deflections of an electron as it slows down from $E_{0}$ to $E . f_{1}(E)$ and $f_{2}(E)$ have the following common properties.

(1) They both equal 0 at $E=E_{0}$ and monotonically increase to their maximum values as $E$ decreases to 0 .

(2) They are small if and only if the total mean-squared angular deflection of electrons from their initial direction is small. (This always occurs for $E \approx E_{0}$.)

(3) They are large if and only if the total mean-squared angular deflection of electrons from their initial direction is large. (This may occur for $E \approx 0$.)

We will now show the following: for small angular deflections and only small-angle scattering, Eqs. (D1) and (D7)-(D9) reduce to the transverse diffusion equation satisfied by the Fermi-Eyges solution. Also, for large angular deflections, Eqs. (D1) and (D7)-(D9) reduce to the FermiAge equation. 
First, let us consider the limit of small angular deflections ( $f_{1}$ and $f_{2}$ small). Expanding Eqs. (D7)-(D9) in this limit, we obtain

$$
\begin{aligned}
& \mathscr{D}_{r}(E)=\frac{1}{3} \int_{E}^{E_{0}} \frac{d E^{\prime}}{S\left(E^{\prime}\right)}\left[f_{2}\left(E^{\prime}\right)+O\left(f^{2}\right)\right], \\
& \mathscr{D}_{z}(E)=2 \int_{E}^{E_{0}} \frac{d E^{\prime}}{S\left(E^{\prime}\right)}\left(f_{1}\left(E^{\prime}\right)-\frac{1}{3} f_{2}\left(E^{\prime}\right)+O\left(f^{2}\right)\right),
\end{aligned}
$$

$$
v(E) \approx 1 .
$$

For the case of negligible large-angle scattering, $\Sigma_{s}\left(E, \mu_{0}\right)$ is a very highly peaked function of $\mu_{0}$ near $\mu_{0}=1$. Thus, by Eq. (7),

$$
\begin{aligned}
\Sigma_{a 2}(E) & =2 \pi \int_{-1}^{1} d \mu_{0}\left[1-P_{2}\left(\mu_{0}\right)\right] \Sigma_{s}\left(E, \mu_{0}\right) \\
& =2 \pi \int_{-1}^{1} d \mu_{0}\left(\frac{3}{2}\left(1+\mu_{0}\right)\left(1-\mu_{0}\right)\right) \Sigma_{s}\left(E, \mu_{0}\right) \\
& \approx 2 \pi \int_{-1}^{1} d \mu_{0}\left[3\left(1-\mu_{0}\right)\right] \Sigma_{s}\left(E, \mu_{0}\right) \\
& =3 \Sigma_{a 1}(E)=\frac{3}{2} T(E) .
\end{aligned}
$$

Hence, $f_{2}(E) \approx 3 f_{1}(E)$, and with $O\left(f^{2}\right)$ error, Eqs. (D13) and (D14) reduce to

$$
\begin{aligned}
& \mathscr{D}_{r}(E)=\frac{1}{2} \int_{E}^{E_{0}} \frac{d E^{\prime}}{S\left(E^{\prime}\right)} \int_{E^{\prime}}^{E_{0}} d E^{\prime \prime} \frac{T\left(E^{\prime \prime}\right)}{S\left(E^{\prime \prime}\right)}, \\
& \mathscr{D}_{z}(E)=0 .
\end{aligned}
$$

Now we introduce Eqs. (D15), (D17), and (D18) into Eq. (D4); the resulting equation implies $E=E(z)$, with

$$
\frac{d E}{S(E)}=-d z
$$

Using this result to eliminate $E$ as an independent variable, we obtain exactly the "transverse" diffusion equation satisfied by the Fermi-Eyges solution.

This shows that in the limit of small angular deflections $\left(f_{1}\right.$ and $f_{2}$ small) and no large-angle scattering, the MM drift diffusion equation for $F$ limits to the transverse diffusion equation satisfied by the Fermi-Eyges solution.

Next, we consider the limit of large angular deflections ( $f_{1}$ and $f_{2}$ large), Eqs. (D7)-(D9) yield

$$
\begin{aligned}
& \mathscr{D}_{r}(E) \approx \mathscr{D}_{z}(E) \approx \frac{e^{-f_{1}(E)}}{3} \int_{E}^{E_{0}} \frac{d E^{\prime}}{S\left(E^{\prime}\right)} e^{f_{1}\left(E^{\prime}\right)}, \\
& v(E) \approx 0 .
\end{aligned}
$$

The integral in Eq. (D20) is dominated by values of $E^{\prime}$ near $E^{\prime}=E$. Therefore,

$$
\begin{aligned}
\int_{E}^{E_{0}} & \frac{d E^{\prime}}{S\left(E^{\prime}\right)} e^{f_{1}\left(E^{\prime}\right)} \\
& =-2 \int_{E}^{E_{0}} \frac{d E^{\prime}}{T\left(E^{\prime}\right)} e^{f_{1}\left(E^{\prime}\right)} \frac{d f_{1}\left(E^{\prime}\right)}{d E^{\prime}} d E^{\prime} \\
& \approx-\frac{2}{T(E)} \int_{E}^{E_{0}} d E^{\prime} e^{f_{1}\left(E^{\prime}\right)} \frac{d f_{1}\left(E^{\prime}\right)}{d E^{\prime}} d E^{\prime} \\
& =\frac{2}{T(E)}\left[e^{f_{1}(E)}-1\right],
\end{aligned}
$$

so Eq. (D20) yields

$$
\mathscr{D}_{r}(E) \approx \mathscr{D}_{z}(E) \approx \frac{2}{3 T(E)}
$$

Introducing Eqs. (D21) and (D23) into Eq. (D1), we obtain the Fermi-Age equation.

This shows that in the limit of large angular deflections ( $f_{1}$ and $f_{2}$ large), the $\mathrm{MM}$ drift diffusion equation for $F$ limits to the Fermi age equation. Depending on the scattering properties of the target material, the maximum values of $f_{1}$ and $f_{2}$ may or may not become large enough for Fermi-Age theory to apply. If $f_{1}$ and $f_{2}$ do become sufficiently "large,", then electrons will "diffuse" far away from their initial direction and become nearly isotropic in their angular distribution; this is the situation in which Fermi-Age theory becomes valid. Eq. (32) and Figs. 5 and 6 show that for 10 and $20 \mathrm{MeV}$ electrons in water, $f_{1}$ (and $f_{2}$ ) do become very large as $E \rightarrow 0$. Therefore, in such beams, Fermi-Age theory does become a valid approximation for small electron energies.

${ }^{a}$ Current address: THREE T, Inc., Ann Arbor, Michigan 48105.

${ }^{1}$ K. R. Hogstrom, M. D. Mills, and P. R. Almond, 'Electron beam dose calculations,"' Phys. Med. Biol. 26, 445-459 (1981).

${ }^{2}$ A. Brahme, I. Lax, and P. Andreo, "Electron beam dose planning using discrete Gaussian beams,' Acta Radiol. Oncol. 20, 147-158 (1981).

${ }^{3}$ B. L. Werner, F. M. Kahn, and F. C. Deibel, "A model for calculating electron beam scattering in treatment planning,' Med. Phys. 9, 180-187 (1982).

${ }^{4}$ B. Rossi and K. Griesen, "Cosmic ray theory,' Rev. Mod. Phys. 13, 240-309 (1941). (Fermi's work is cited on pp. 265-268.)

${ }^{5}$ L. Eyges, "Multiple scattering with energy loss,' Phys. Rev. 74, 15341535 (1948).

${ }^{6}$ I. Lax, A. Brahme, and P. Andreo, "Electron beam dose planning using Gaussian beams: Improved radial dose profiles,' Acta Radiol. Suppl. Stockholm 364, 49-59 (1983).

${ }^{7}$ D. Jette, A. Pagnamenta, L. H. Lanzl, and M. Rozenfeld, "The application of multiple scattering theory to therapeutic electron dosimetry," Med. Phys. 10, 141-146 (1983).

${ }^{8}$ A. Brahme, "Current algorithms for computed electron beam dose planning,' Radiother. Oncol. 3, 347-362 (1985).

${ }^{9}$ D. Jette, "Electron dose calculation using multiple-scattering theory. A. Gaussian multiple-scattering theory,', Med. Phys. 15, 123-137 (1988).

${ }^{10} \mathrm{H}$. Huizenga, 'Electron beam dose calculation algorithms review,' Proceedings of The 10th International Conference on The Use of Computers in Radiation Therapy, edited by S. Hukku and P. S. Iyer (Lucknow, India, 1990), pp. 24-28.

${ }^{11}$ D. Jette and A. Bielajew, "Electron dose calculation using multiplescattering theory: Second-order multiple-scattering theory,' Med. Phys. 16, 698-711 (1989).

${ }^{12}$ P. R. M. Storchi and H. Huizenga, "On a numerical approach of the pencil beam model,', Phys. Med. Biol. 30, 467-473 (1985). 
${ }^{13}$ I. A. D. Bruinvis, W. A. F. Mathol, and P. Andreo, "'Inclusion of electron range straggling in the Fermi-Eyges multiple-scattering theory," Phys. Med. Biol. 34, 491-507 (1989).

${ }^{14}$ A. S. Shiu and K. R. Hogstrom, "Pencil-beam redefinition algorithm for electron dose distributions,'” Med. Phys. 18, 7-18 (1991).

${ }^{15}$ C. X. Yu, W. S. Ge, and J. W. Wong, "A multiray model for calculating electron pencil beam distribution,'” Med. Phys. 15, 662-671 (1988).

${ }^{16}$ H. Huizenga and P. R. M. Storchi, "Numerical calculation of energy deposition by broad high energy electron beams,' Phys. Med. Biol. 34, 1371-1396 (1989).

${ }^{17}$ H. Neuenschwander, T. R. Mackie, and P. J. Reckwerdt, " $\mathrm{MMC}-\mathrm{A}$ high-performance Monte Carlo code for electron beam treatment planning,' Phys. Med. Biol. 40, 543-574 (1995).

${ }^{18}$ H. W. Lewis, "Multiple scattering in an infinite medium,' Phys. Rev. 78, 526-529 (1950).

${ }^{19}$ L. V. Spencer and U. Fano, "'Penetration and diffusion of X-rays. Calculation of spatial distributions by polynomial expansion,', Phys. Rev. 81, 464-466 (1951).

${ }^{20}$ L. V. Spencer and U. Fano, "Penetration and diffusion of x-rays. Calculation of spatial distributions by polynomial expansion,', J. Res. Natl. Bur. Stand. 46, 446-456 (1951).

${ }^{21}$ L. V. Spencer, "'Theory of electron penetration,' Phys. Rev. 98, 15971615 (1955)

${ }^{22}$ U. Fano, L. V. Spencer, and M. J. Berger, "Penetration and diffusion of X-rays,' Handbuch der Physik, edited by S. Flügge (Springer-Verlag, Berlin, 1959), Vol. 38/2.

${ }^{23}$ N. D. Kessaris, "Penetration of high-energy electron beams in water," Phys. Rev. 145, 164-174 (1966).

${ }^{24}$ J. K. Shultis and R. E. Faw, Radiation Shielding (Prentice-Hall PTR, Upper Saddle River, NJ, 1996), p. 386.

${ }^{25}$ M. M. R. Williams, "The role of the Boltzmann transport equation in radiation damage calculations,', Prog. Nucl. Energy 3, 1-65 (1979).
${ }^{26}$ S. C. Klevenhagen, Physics of Electron Beam Therapy, Medical Physics Handbooks (Adam Hilger, Bristol, 1985), Vol. 13.

${ }^{27}$ A. Z. Akcasu and E. W. Larsen, "A statistical model for electron transport and dose calculations,' Ann. Nucl. Energy 23, 253-267 (1996).

${ }^{28}$ W. R. Nelson, H. Hirayama, and D. W. O. Rogers, "The EGS4 code system,' Stanford Linear Accelerator Center Publication No. 265, 1985.

${ }^{29}$ A. F. Bielajew and D. W. O. Rogers, "PRESTA: The Parameter Reduced Electron-Step Transport Algorithm for electron Monte Carlo transport,', Nucl. Instrum. Methods B 18, 165-181 (1987).

${ }^{30}$ L. J. Lorence, Jr., J. E. Morel, and G. D. Valdez, “User's Guide to CEPXS/ONEDANT. A One-Dimensional Coupled Electron-Photon Discrete Ordinates Code package,' Sandia National Laboratory Report No. SAND 89-1661, 1989.

${ }^{31}$ C. Börgers and E. W. Larsen, "On the accuracy of the Fokker-Planck and Fermi pencil beam equations for charged particle transport,' Med. Phys. 23, 1749-1759 (1996).

${ }^{32}$ S. Chandresekhar, "Stochastic problems in physics and astronomy," Rev. Mod. Phys. 15, 1-89 (1943).

${ }^{33}$ G. C. Pomraning, "The Fokker-Planck operator as an asymptotic limit," Math. Mod. Methods Appl. Sci. 2, 21-36 (1992).

${ }^{34} \mathrm{M}$. Caro and J. Ligou, "Treatment of scattering anisotropy of neutrons through the Boltzmann-Fokker-Planck equation,' Nucl. Sci. Eng. 83, 242-252 (1983).

${ }^{35}$ M. Landesman and J. E. Morel, “Angular Fokker-Planck decomposition and representation techniques,'” Nucl. Sci. Eng. 103, 1-11 (1989).

${ }^{36}$ R. C. Weast, S. M. Selby, and C. D. Hodgman, Handbook of Mathematical Tables, 2nd ed. (Chemical Rubber Co., Cleveland, 1964).

${ }^{37}$ A. R. Edmonds, Angular Momentum in Quantum Mechanics (Princeton University Press, Princeton, 1957).

${ }^{38}$ S. Goudsmit and J. L. Saunderson, "Multiple scattering of electrons," Phys. Rev. 57, 24 (1940)

${ }^{39}$ L. M. Delves and J. L. Mohamed, Computational Methods for Integral Equations (Cambridge University Press, Cambridge, 1988). 